\title{
Klein-Gordon Equations on Modulation Spaces
}

\author{
Guoping Zhao, ${ }^{1}$ Jiecheng Chen, ${ }^{2}$ and Weichao $\mathrm{Guo}^{3}$ \\ ${ }^{1}$ Department of Mathematics, Zhejiang University, Hangzhou 310027, China \\ ${ }^{2}$ Department of Mathematics, Zhejiang Normal University, Jinhua 321004, China \\ ${ }^{3}$ Department of Mathematics, Xiamen University, Xiamen 361005, China \\ Correspondence should be addressed to Weichao Guo; maodunguo@163.com
}

Received 16 January 2014; Accepted 26 April 2014; Published 20 May 2014

Academic Editor: Simeon Reich

Copyright (C) 2014 Guoping Zhao et al. This is an open access article distributed under the Creative Commons Attribution License, which permits unrestricted use, distribution, and reproduction in any medium, provided the original work is properly cited.

We consider the Cauchy problem for a family of Klein-Gordon equations with initial data in modulation spaces $M_{p, 1}^{a}$. We develop the well-posedness, blowup criterion, stability of regularity, scattering theory, and stability theory.

\section{Introduction}

In this paper, we consider the Cauchy problem for the following nonlinear Klein-Gordon equation in the space $\mathbb{R} \times$ $\mathbb{R}^{n}=\mathbb{R}_{t} \times \mathbb{R}_{x}^{n}$ :

$$
\begin{gathered}
u_{t t}+(I-\Delta) u+f(u)=0, \\
u\left(t_{0}, x\right)=u_{0}, \\
u_{t}\left(t_{0}, x\right)=u_{1},
\end{gathered}
$$

where $u(t, x)$ is a complex-valued function in $I \times \mathbb{R}^{n}$ for some time interval $I$ containing $t_{0}$, the initial data $\left(u_{0}, u_{1}\right)$ lies in the product of modulation spaces $M_{p, 1}^{s} \times M_{p, 1}^{s-1}(1 \leq p \leq$ $\infty, s \geq 0)$, and the nonlinear term $f(u)=\pi\left(u^{k+1}\right)$ is any $(k+1)$-time product of $u$ and $\bar{u}, k \in \mathbb{N}$. To understand this research problem and its historical developments, the reader may see Ruzhansky et al. [1] for a brief survey of nonlinear evolution equations on the modulation spaces. Concerning the well-posedness of solution to the Schrödinger equation in the modulation space, readers can refer to $[2,3]$.

We give some remarks about our results. The known study of the Klein-Gordan equations (or other dispersive equations) on modulation spaces must be based on the assumption that the nonlinear term $f(u)$ is a polynomial. This assumption is also necessary in this paper; in fact, this is an open problem that if

$$
\left\||u|^{\lambda} u\right\|_{M_{p, 1}} \leq C\|u\|_{M_{p, 1}^{\lambda}}^{\lambda}\|u\|_{M_{p, 1}}
$$

holds for any positive real constant $\lambda$.

We recall that $s_{k}=n / 2-2 / k$ is the critical index for (1). Up to now, we cannot solve (1) in $H^{s}$ for the case that $s<s_{k}$ (the sup-critical case). On the other hand, we notice that the modulation space $M_{p, 1}$ has low regularity property. More precisely, for sufficiently large $s$, we have the following embedding:

$$
H^{s} \subset M_{p, 1} \text {. }
$$

In other words, the modulation space $M_{p, q}$ has lower regularity than $H^{s}$ for large $s$. So, for large $s_{k}$ (high dimension for instance), one can solve (1) in $M_{p, 1}^{s}$ which contains supcritical initial dates in $H^{s}$ for $s<s_{k}$.

The local well-posedness of (1) in $M_{p, 1}^{s}(1 \leq p \leq \infty$, $s \geq 0$ ) is a result of Bényi and Okoudjou [4]; see also Wang [2] for a global result with small initial data. These results say that, for $\left(u_{0}, u_{1}\right) \in M_{p, 1}^{s} \times M_{p, 1}^{s-1}$, there exists a positive $T=T\left(\left\|u_{0}\right\|_{M_{p, 1}^{s}},\left\|u_{1}\right\|_{M_{p, 1}^{s-1}}\right) \leq \infty$ such that (1) has a unique solution $u(t, x) \in C\left([0, T], M_{p, 1}^{s}\right)$. Moreover, the lifetime of the solution can be proved to be bounded below by a decreasing positive function $g$ depending on $\left\|u_{0}\right\|_{M_{p, 1}^{s}},\left\|u_{1}\right\|_{M_{p, 1}^{s-1}}$ t that is, $T\left(u_{0}, u_{1}\right) \geq g\left(\left\|u_{0}\right\|_{M_{p, 1}^{s}},\left\|u_{1}\right\|_{M_{p, 1}^{s-1}}\right)$. It 
also asserts that if a strong $M_{p, 1}^{s}$ solution keeps its $M_{p, 1}^{s}$ norm bounded in a bounded interval, it can be extended beyond the endpoint. Hence, the following blowup criterion holds:

$$
T<\infty \text { implies } \lim _{t \rightarrow T}\|u(t)\|_{M_{p, 1}^{s}}=\infty .
$$

In this paper, we will develop a stronger blowup criterion which says that a blowup solution cannot blow up too slowly (see Corollary 8 and Remark 9). We also study the regularity of solutions and show that the regularity is stable along the lifetime. As an application, the global existence of low regularity ensures the global existence of high regularity.

Compared with $C\left(I, M_{p, 1}^{s}\right)$ (used in [4]), the space $L_{t}^{r}\left(I, M_{p, 1}^{s}\right)$ seems more suitable for applying continuity argument, which is the key point for obtaining the perturbation theorem, especially the long-time version. So we choose $L_{t}^{r}\left(I, M_{p, 1}^{s}\right)$ as our work space and establish the nonlinear estimate associated with this work space in Section 2.

In Section 3, we will establish the local theory. We first use the fixed point theorem to construct a local-in-time solution $u \in L_{t}^{r}\left(I, M_{p, 1}^{s}\right)$ to (1). Then, we verify that such solution is a strong $M_{p, 1}^{s}$ solution in the sense that $u \in C\left(I, M_{p, 1}^{s}\right) \cap$ $C^{1}\left(I, M_{p, 1}^{s-1}\right)$ and is unique in the category of strong solution. Finally, we study the regularity of solutions and deduce a stronger blowup criterion which implies the high rate of blowup. We will develop the scattering results in Section 4. In Section 5, we establish a stability theory for (1) and obtain the continuous dependence as a corollary.

Denote the operator $\omega=\mathrm{Id}-\Delta$, and

$$
\varphi(\omega):=\mathscr{F}^{-1} \varphi\left((1+|\xi|)^{2}\right) \mathscr{F}
$$

for any function $\varphi$. Using this notation, we define

$$
K(t):=\frac{\sin \left(t \omega^{1 / 2}\right)}{\omega^{1 / 2}}
$$

and the Klein-Gordon semigroup:

$$
G(t):=e^{i t \omega^{1 / 2}} .
$$

We now state our main results. Unless otherwise specified, we assume that the letters $k, n$ are integers such that $1 /(k+2)+1 / n(k+1)<1 / 2$ and $(p, r)$ is $k$-admissible (Definition 25). First, we have the following local theorem.

Theorem 1 (local well-posedness). Let I be a compact time interval that contains $t_{0}$. Let $\left(u_{0}, u_{1}\right) \in M_{p, 1}^{s} \times M_{p, 1}^{s-1}$ satisfy

$$
\left\|K^{\prime}\left(t-t_{0}\right) u_{0}+K\left(t-t_{0}\right) u_{1}\right\|_{L_{t}^{r}\left(I, M_{p, 1}^{s}\right)} \leq \eta
$$

for some $0<\eta \leq \eta_{0}$, where $\eta_{0}$ is a small constant (depending only on $n, k)$. Then, there exists a unique solution $u \in$ $L_{t}^{r}\left(I, M_{p, 1}^{s}\right)$ to (1). Moreover, $u$ is a $M_{p, 1}^{s}$ strong solution to (1) in the sense that $u \in C\left(I, M_{p, 1}^{s}\right) \cap C^{1}\left(I, M_{p, 1}^{s-1}\right)$, and one also has

$$
\|u\|_{L_{t}^{r}\left(I, M_{p, 1}^{s}\right)} \leq 2 \eta
$$

From Lemma 19, we can verify the condition (8) by choosing $I$ sufficiently small. So this theorem already gives local existence for large $\left\|u_{0}\right\|_{M_{p, 1}^{s}},\left\|u_{1}\right\|_{M_{p, 1}^{s-1}}$ data. On the other hand, by inequality (63), we have the following global result as an application.

Corollary 2 (global well-posedness for small fine data). Let $2 \sigma(p)=(n+2)(1 / 2-1 / p)$. Assume that $\left(u_{0}, u_{1}\right) \in M_{p^{\prime}, 1}^{s+2 \sigma(p)} \times$ $M_{p^{\prime}, 1}^{s+2 \sigma(p)-1}$ satisfies

$$
\left\|u_{0}\right\|_{M_{p^{\prime}, 1}^{s+2 \sigma(p)}}+\left\|u_{1}\right\|_{M_{p^{\prime}, 1}^{s+2 \sigma(p)-1}} \leq \delta_{0}
$$

for some small constant $\delta_{0}>0$. Then, there exists a unique global solution $u \in L_{t}^{r}\left(\mathbb{R}, M_{p, 1}^{s}\right)$. Similarly, $u$ is also a strong solution; that is, $u \in C\left(\mathbb{R}, M_{p, 1}^{s}\right) \cap C^{1}\left(\mathbb{R}, M_{p, 1}^{s-1}\right)$. One also has the bound

$$
\|u\|_{L_{t}^{r}\left(\mathbb{R}, M_{p, 1}^{s}\right)} \lesssim\left\|u_{0}\right\|_{M_{p^{\prime}, 1}^{s+2 \sigma(p)}}+\left\|u_{1}\right\|_{M_{p^{\prime}, 1}^{s+2 \sigma(p)-1}}
$$

More precisely, we have the following global wellposedness result which gives the decay rate of solutions.

Corollary 3 (another form of global well-posedness for small fine data). Assume that $\left(u_{0}, u_{1}\right) \in M_{p^{\prime}, 1}^{s+2 \sigma(p)} \times M_{p^{\prime}, 1}^{s+2 \sigma(p)-1}$ satisfies

$$
\left\|u_{0}\right\|_{M_{p^{\prime}, 1}^{s+2 \sigma(p)}}+\left\|u_{1}\right\|_{M_{p^{\prime}, 1}^{s+2 \sigma(p)-1}}<\delta
$$

for some small constant $\delta>0$. Then, there exists a unique global solution $u$ :

$$
u \in X:=\left\{u: \sup _{t \in \mathbb{R}}(1+|t|)^{n(1 / 2-1 / p)}\|u\|_{M_{p, 1}^{s}}<\infty\right\} .
$$

In the proof of Theorem 1, uniqueness is an immediate conclusion by the fixed point theorem. But, in fact, we have the following stronger result.

Theorem 4 (unconditional uniqueness in $M_{p, 1}^{s}$ ). Let $I$ be a time interval containing $t_{0}, p \in[1, \infty], k \in \mathbb{N}$, and let $u, v \in$ $C\left(I, M_{p, 1}^{s}\right)$ be two strong solutions to (1) in the sense of (45) with the same initial data $u\left(t_{0}\right)=v\left(t_{0}\right), u_{t}\left(t_{0}\right)=v_{t}\left(t_{0}\right)$; then $u=v$ in $M_{p, 1}^{s}$ for all $t \in I$.

By combining the above uniqueness result with the local theorem, one can define the maximal interval $I$ of the strong solution; thus, we have the following standard blowup criterion.

Theorem 5 (blowup criterion). Let $u_{0} \in M_{p, 1}^{s}, u_{1} \in M_{p, 1}^{s-1}$, and let $I=\left(T_{\min }, T_{\max }\right)$ be the maximal interval. If $T_{\max }<$ $\infty\left(T_{\min }>-\infty\right)$, then one has

$$
\|u\|_{L_{t}^{r}\left(\left[t_{0}, T_{\max }\right], M_{p, 1}^{s}\right)}=\infty\left(\|u\|_{L_{t}^{r}\left(\left[T_{\min }, t_{0}\right], M_{p, 1}^{s}\right)}=\infty\right) .
$$

The above blowup criterion will be improved soon as an application of Lemmas 31 and 32. For completeness, we also give a proof for this weak version. Then, we give a regularity result. 
Theorem 6 (persistence of regularity). Let $1 \leq p_{1}, p_{2} \leq \infty$, $0 \leq s_{1}, s_{2}<\infty$, and $k \in \mathbb{N}$, and letu be a strong $M_{p_{2}, 1}^{s_{2}}$ solution to (1) with its maximal existence interval I. If $u\left(t_{0}\right) \in M_{p_{1}, 1}^{s_{1}}$ and $u_{t}\left(t_{0}\right) \in M_{p_{1}, 1}^{s_{1}-1}$ for some $t_{0} \in I$, then $u$ is also a strong $M_{p_{1}, 1}^{s_{1}}$ solution with the same maximal interval.

Remark 7. Combining the above theorem with global result in $M_{2,1} \times M_{2,1}^{-1}$ in [2], one can easily get the global wellposedness in $M_{p, 1}^{s} \times M_{p, 1}^{s-1}$ with small initial data for $p \in[1,2]$ and $s \geq 0$.

Thus, it is not possible to develop a singularity which causes the $M_{p_{1}, 1}^{s_{1}} \times M_{p_{1}, 1}^{s_{1}-1}$ norm to blow up while the $M_{p_{2}, 1}^{s_{2}} \times$ $M_{p_{2}, 1}^{s_{2}-1}$ norm remains bounded. We also see that the regularity is stable, because if a solution lies in $C\left(I, M_{p_{2}, 1}^{s_{2}}\right)$ and is not in $M_{p_{1}, 1}^{s_{1}} \times M_{p_{1}, 1}^{s_{1}-1}$ at some initial time $t_{0}$, it never belongs to $M_{p_{1}, 1}^{s_{1}} \times M_{p_{1}, 1}^{s_{1}-1}$ at any later (or earlier) time. As an application (see also Lemmas 31 and 32), we have the following stronger version of blowup criterion.

Corollary 8 (stronger blowup criterion). Let $p \in[1, \infty], k \in$ $\mathbb{N}$, and a strong solution of Cauchy problem (1) blows up in a finite time $T_{\max }<\infty\left(T_{\min }>-\infty\right)$ if and only if

$$
\|u(t)\|_{L_{t}^{k}\left(\left[t_{0}, T_{\max }\right], M_{\infty, 1}^{0}\right)}=\infty\left(\|u(t)\|_{L_{t}^{k}\left(\left[T_{\min }, t_{0}\right], M_{\infty, 1}^{0}\right)}=\infty\right) .
$$

Remark 9. From another point of view, the above blowup criterion implies that $\|u(t)\|_{M_{p, 1}^{s}}$ cannot blow up too slowly when $t$ tends to a finite blowup time $T$; that is,

$$
\varlimsup_{t \rightarrow T} \frac{\|u(t)\|_{M_{p, 1}^{s}}}{|T-t|^{-1 / k+\varepsilon}}=+\infty
$$

for every $\mathcal{\varepsilon}>0$.

We also obtain a scattering theorem for these equations provided a bounded $L_{t}^{r}\left(\mathbb{R}, M_{p, 1}^{s}\right)$ norm.

Theorem $10\left(L_{t}^{r}\left(\mathbb{R}, M_{p, 1}^{s}\right)\right.$ bounds imply scattering $)$. Let $u_{0} \in$ $M_{p, 1}^{s}, u_{1} \in M_{p, 1}^{s-1}$, and let $u$ be a global strong $M_{p, 1}^{s}$ solution to (1) such that

$$
\|u\|_{L_{t}^{r}\left(\mathbb{R}, M_{p, 1}^{s}\right)} \leq M
$$

for some constant $M>0$. Then, there exist $v_{1}^{ \pm}, v_{2}^{ \pm} \in M_{p, 1}^{s}$ such that $v^{ \pm} \triangleq G(t) v_{1}^{ \pm}+G(t) v_{2}^{ \pm}$are solutions to the free Klein-Gordon equation $u_{t t}+(I-\Delta) u=0$, and

$$
\left\|u(t)-v^{ \pm}\right\|_{M_{p, 1}^{s}} \longrightarrow 0
$$

as $t \rightarrow \pm \infty$.

Finally, we will discuss the stability theory. The stability theory for (1) means that given an approximate solution

$$
\tilde{u}_{t t}+(I-\Delta) \tilde{u}=f(\widetilde{u})+e
$$

to (1), with $e$ and $u-\tilde{u}, u_{t}-\tilde{u}_{t}$ small in a suitable space, is it possible to show that the genuine solution $u$ to (1) stays very close to $\tilde{u}$ in some sense (for instance, in the $M_{p, 1}^{s}$ )? Note that the question of continuous dependence of the data corresponds to the case $e=0$ and the uniqueness theory to the case $e=0, u\left(t_{0}\right)=\widetilde{u}\left(t_{0}\right)$. We have the following shorttime perturbations and long-time perturbations.

Theorem 11 (short-time perturbations). Let I be a compact time interval, and let $\tilde{u}$ be an approximate solution to (1) in the sense of (19). Assume that $\tilde{u}$ has a uniform $M_{p, 1}^{s}$ bound:

$$
\|\widetilde{u}\|_{L_{t}^{\infty}\left(I, M_{p, 1}^{s}\right)} \leq M, \quad\left\|\widetilde{u}_{t}\right\|_{L_{t}^{\infty}\left(I, M_{p, 1}^{s-1}\right)} \leq \widetilde{M}
$$

for some constant $M, \widetilde{M}>0$. Let $t_{0} \in I$ and let $u\left(t_{0}\right) \in M_{p, 1}^{s}$, $u_{t}\left(t_{0}\right) \in M_{p, 1}^{s-1}$ be close to $\widetilde{u}\left(t_{0}\right), \widetilde{u}_{t}\left(t_{0}\right)$, respectively, in the sense that

$$
\left\|u\left(t_{0}\right)-\widetilde{u}\left(t_{0}\right)\right\|_{M_{p, 1}^{s}} \leq M^{\prime}, \quad\left\|u_{t}\left(t_{0}\right)-\widetilde{u}_{t}\left(t_{0}\right)\right\|_{M_{p, 1}^{s-1}} \leq \widetilde{M^{\prime}}
$$

for some $M^{\prime}, \widetilde{M^{\prime}}>0$. Moreover, assume the following smallness conditions:

$$
\begin{gathered}
\|\widetilde{u}\|_{L_{t}^{r}\left(I, M_{p, 1}^{s}\right)} \leq \varepsilon_{c}, \\
\| K^{\prime}\left(t-t_{0}\right)\left(u\left(t_{0}\right)-\widetilde{u}\left(t_{0}\right)\right) \\
+K\left(t-t_{0}\right)\left(u_{t}\left(t_{0}\right)-\widetilde{u}_{t}\left(t_{0}\right)\right) \|_{L_{t}^{r}\left(I, M_{p, 1}^{s}\right)} \leq \varepsilon, \\
\|e\|_{L_{t}^{r /(k+1)}\left(I, M_{p^{\prime}, 1}^{s}\right)} \leq \varepsilon,
\end{gathered}
$$

for some $0<\varepsilon<\varepsilon_{c}$, where $\varepsilon_{c}$ is a small constant.

Then, there exists a solution $u$ to (1) with initial values $u\left(t_{0}\right), u_{t}\left(t_{0}\right)$ at time $t_{0}$ satisfying

$$
\begin{gathered}
\left\|\left(\partial_{t}^{2}+I-\Delta\right)(u-\widetilde{u})+e\right\|_{L_{t}^{r /(k+1)}\left(I, M_{p l, 1}^{s}\right)} \lesssim \varepsilon, \\
\|u-\widetilde{u}\|_{L_{t}^{r}\left(I, M_{p, 1}^{s}\right)} \lesssim \varepsilon \\
\|u-\widetilde{u}\|_{L_{t}^{\infty}\left(I, M_{p, 1}^{s}\right)} \lesssim C(|I|)\left(M^{\prime}+\widetilde{M^{\prime}}+\varepsilon\right), \\
\left\|u_{t}-\widetilde{u}_{t}\right\|_{L_{t}^{\infty}\left(I, M_{p, 1}^{s-1}\right)} \lesssim C(|I|)\left(M^{\prime}+\widetilde{M^{\prime}}+\varepsilon\right), \\
\left\|u_{t}\right\|_{L_{t}^{\infty}\left(I, M_{p, 1}^{s}\right)} \lesssim C(|I|)\left(M+M^{\prime}+\widetilde{M^{\prime}}+\varepsilon\right), \\
\left\|u_{t}\right\|_{L_{t}^{\infty}\left(I, M_{p, 1}^{s-1}\right)} \lesssim C(|I|)\left(\widetilde{M}+M^{\prime}+\widetilde{M^{\prime}}+\varepsilon\right) .
\end{gathered}
$$

Theorem 12 (long-time perturbations). Let I be a compact time interval, and let $\tilde{u}$ be an approximate solution to (1) in the sense of (19) for some function e. Assume that

$$
\begin{gathered}
\|\widetilde{u}\|_{L_{t}^{r}\left(I, M_{p, 1}^{s}\right)} \leq L, \\
\|\widetilde{u}\|_{L_{t}^{\infty}\left(I, M_{p, 1}^{s}\right)} \leq M, \quad\left\|\widetilde{u}_{t}\right\|_{L_{t}^{\infty}\left(I, M_{p, 1}^{s-1}\right)} \leq \widetilde{M}
\end{gathered}
$$


for some constants $M, \widetilde{M}$, and L. Let $t_{0} \in I$ and let $u\left(t_{0}\right) \in$ $M_{p, 1}^{s}, u_{t}\left(t_{0}\right) \in M_{p, 1}^{s-1}$ be close to $\tilde{u}\left(t_{0}\right), \tilde{u}_{t}\left(t_{0}\right)$, respectively, in the sense that

$$
\begin{aligned}
& \left\|u\left(t_{0}\right)-\widetilde{u}\left(t_{0}\right)\right\|_{M_{p, 1}^{s}} \leq M^{\prime}, \\
& \left\|u_{t}\left(t_{0}\right)-\widetilde{u}_{t}\left(t_{0}\right)\right\|_{M_{p, 1}^{s-1}} \leq \widetilde{M^{\prime}}
\end{aligned}
$$

for some $M^{\prime}, \widetilde{M^{\prime}}>0$. Moreover, assume the following smallness conditions:

$$
\begin{aligned}
& \| K^{\prime}\left(t-t_{0}\right)\left(u\left(t_{0}\right)-\widetilde{u}\left(t_{0}\right)\right) \\
& +K\left(t-t_{0}\right)\left(u_{t}\left(t_{0}\right)-\widetilde{u}_{t}\left(t_{0}\right)\right) \|_{L_{t}^{r}\left(I, M_{p, 1}^{s}\right)} \leq \varepsilon \\
& \|e\|_{L_{t}^{r /(k+1)}\left(I, M_{p^{\prime}, 1}^{s}\right)} \leq \varepsilon
\end{aligned}
$$

for some $0<\varepsilon<\varepsilon_{1}$, where $\varepsilon_{1}=\varepsilon_{1}(L)$ is a small constant. Then, there exists a solution $u$ to (1) with initial values $u\left(t_{0}\right), u_{t}\left(t_{0}\right)$ at time $t_{0}$ satisfying

$$
\begin{gathered}
\left\|\left(\partial_{t}^{2}+I-\Delta\right)(u-\widetilde{u})+e\right\|_{L_{t}^{r /(k+1)}\left(I, M_{p^{\prime}, 1}^{s}\right)} \leqslant C(L) \varepsilon, \\
\|u-\widetilde{u}\|_{L_{t}^{r}\left(I, M_{p, 1}^{s}\right)} \leqslant C(L) \varepsilon \\
\|u-\widetilde{u}\|_{L_{t}^{\infty}\left(I, M_{p, 1}^{s}\right)} \leqslant C(|I|, L)\left(M^{\prime}+\widetilde{M^{\prime}}+\varepsilon\right), \\
\left\|u_{t}-\widetilde{u}_{t}\right\|_{L_{t}^{\infty}\left(I, M_{p, 1}^{s-1}\right)} \leqslant C(|I|, L)\left(M^{\prime}+\widetilde{M^{\prime}}+\varepsilon\right), \\
\|u\|_{L_{t}^{\infty}\left(I, M_{p, 1}^{s}\right)} \lesssim C(|I|, L)\left(M+M^{\prime}+\widetilde{M^{\prime}}+\varepsilon\right), \\
\left\|u_{t}\right\|_{L_{t}^{\infty}\left(I, M_{p, 1}^{s-1}\right)} \lesssim C(|I|, L)\left(\widetilde{M}+M^{\prime}+\widetilde{M^{\prime}}+\varepsilon\right) .
\end{gathered}
$$

As applications of the above stability theorems, we have the following corollaries.

Corollary 13 (continuous dependence). Assume that $1 /(k+$ $2) \leq 1 / p<1 / 2-1 / n(k+1)$, and $u(t)$ is a strong solution to (1) with initial data $u\left(t_{0}\right)=u_{0} \in M_{p, 1}^{s}, u_{t}\left(t_{0}\right)=u_{1} \in M_{p, 1}^{s-1}$. If $\left(u_{0, n}, u_{1, n}\right) \rightarrow\left(u_{0}, u_{1}\right)$ in $M_{p, 1}^{s} \times M_{p, 1}^{s-1}$, then

$$
\begin{aligned}
& \varliminf_{n \rightarrow \infty} T_{\max }\left(u_{0, n}, u_{1, n}\right) \geq T_{\max }\left(u_{0}, u_{1}\right), \\
& \varlimsup_{n \rightarrow \infty} T_{\min }\left(u_{0, n}, u_{1, n}\right) \leq T_{\min }\left(u_{0}, u_{1}\right) .
\end{aligned}
$$

For every compact interval $I \subset\left(T_{\min }\left(u_{0}, u_{1}\right), T_{\max }\left(u_{0}, u_{1}\right)\right)$, let $u_{n}$ be the solution to (1) on I with initial $u_{0, n}, u_{1, n}$, and then we have

$$
\begin{aligned}
& \left\|u_{n}-u\right\|_{L_{t}^{\infty}\left(I, M_{p, 1}^{s}\right)} \\
& \quad \leq C\left(\left\|u_{0, n}-u_{0}\right\|_{M_{p, 1}^{s}}+\left\|u_{1, n}-u_{1}\right\|_{M_{p, 1}^{s-1}}\right), \\
& \left\|\left(u_{n}\right)_{t}-u_{t}\right\|_{L_{t}^{\infty}\left(I, M_{p, 1}^{s}\right)} \\
& \quad \leq C\left(\left\|u_{0, n}-u_{0}\right\|_{M_{p, 1}^{s}}+\left\|u_{1, n}-u_{1}\right\|_{M_{p, 1}^{s-1}}\right)
\end{aligned}
$$

with $C=C\left(|I|,\|u\|_{L_{t}^{k+1}\left(I, M_{p, 1}^{s}\right)}\right)$; that is, $u_{n} \rightarrow u_{0}$ in $C\left(I, M_{p, 1}^{s}\right) \cap C^{1}\left(I, M_{p, 1}^{s-1}\right)$.

Also, one can deduce continuous dependence for $p \in$ $[1, \infty], k \in \mathbb{N}$ directly without using perturbation theorem, and the proof is not difficult, so we omit the details.

Corollary 14. Assume that $(p, r)$ is a $k$-admissible pair. Denote A by the subset of $M_{p^{\prime}, 1}^{s+2 \sigma(p)} \times M_{p^{\prime}, 1}^{s+2 \sigma(p)-1}$, such that, for every $\left(u_{0}, u_{1}\right) \in A$, the Cauchy problem (1) with initial data $u_{0}, u_{1}$ has a global strong $M_{p, 1}^{s}$ solution $u$ on $\mathbb{R}$ and $\|u\|_{L_{t}^{r}\left(I, M_{p, 1}^{s}\right)}<\infty$. Then, the set $A$ is open in $M_{p^{\prime}, 1}^{s+2 \sigma(p)} \times M_{p^{\prime}, 1}^{s+2 \sigma(p)-1}$.

\section{Preliminaries}

If $X$ and $Y$ are two quantities (typically nonnegative), we will often use the notation $X \lesssim Y$ to denote the statement that $X \leq C Y$ for some absolute constant $C>0$, where $C$ can depend on $n, k, p, r$, but it might be different from line to line. Given $k=\left(k_{1}, k_{2}, \ldots, k_{n}\right) \in \mathbb{Z}^{n}$, we write $|k|:=\left|k_{1}\right|+$ $\left|k_{2}\right|+\cdots+\left|k_{n}\right|,|k|_{\infty}:=\sup _{i=1,2, \ldots, n}\left|k_{i}\right|$, and $\langle k\rangle:=1+|k|$. Let $L_{x}^{r}\left(\mathbb{R}^{n}\right)$ denote the Banach space of functions $f: \mathbb{R}^{n} \rightarrow \mathbb{C}$ whose norm

$$
\|f\|_{L_{x}^{r}\left(\mathbb{R}^{n}\right)}:=\left(\int_{\mathbb{R}^{n}}|f(x)|^{r} d x\right)^{1 / r}<\infty, \quad 1 \leq r<\infty .
$$

The norm $\|f\|_{L_{x}^{\infty}\left(\mathbb{R}^{n}\right)}$ is defined with the usual modification. We also abbreviate $\|\cdot\|_{L_{x}^{r}\left(\mathbb{R}^{n}\right)}$ for $\|\cdot\|_{L^{r}}$, or $\|\cdot\|_{r}$, when there is no confusion. We use $L_{t}^{r}(I, X)$ to denote the space-time norm:

$$
\|u\|_{L_{t}^{r}(I, X)}=\left(\int_{I}\|u\|_{X}^{r} d t\right)^{1 / r}
$$

with the usual modifications when $p, q$, or $r$ is infinite. For the operator $\omega=\operatorname{Id}-\Delta$, the operator

$$
K(t):=\frac{\sin \left(t \omega^{1 / 2}\right)}{\omega^{1 / 2}}
$$

and the Klein-Gordon semigroup

$$
G(t):=e^{i t \omega^{1 / 2}}
$$

have been defined in Section 1. Thus, we may recall Duhamel's formula:

$$
\begin{array}{r}
u(t)=K^{\prime}\left(t-t_{0}\right) u_{0}+K\left(t-t_{0}\right) u_{1} \\
-\int_{t_{0}}^{t} K(t-\tau) f(u(\tau)) d \tau
\end{array}
$$

Also, we recall the integral form of Gronwall's inequality.

Lemma 15 (Gronwall inequality and integral form [5]). Let $A:\left[t_{0}, t_{1}\right] \rightarrow \mathbb{R}^{+}$be continuous and nonnegative, and suppose that A obeys the integral inequality

$$
A(t) \leq C+\int_{t_{0}}^{t} B(s) A(s) d s
$$


for all $t \in\left[t_{0}, t_{1}\right]$, where $C \geq 0$ and $B:\left[t_{0}, t_{1}\right] \rightarrow \mathbb{R}^{+}$is continuous and nonnegative. Then, we have

$$
A(t) \leq C \exp \left(\int_{t_{0}}^{t} B(s) d s\right)
$$

for all $t \in\left[t_{0}, t_{1}\right]$.

Let $\mathcal{S}:=\mathcal{S}\left(\mathbb{R}^{n}\right)$ be the Schwartz space and $\mathcal{S}^{\prime}:=\mathcal{S}^{\prime}\left(\mathbb{R}^{n}\right)$ the tempered distribution space. We introduce the definition of modulation space, which was introduced by Feichtinger [6] in 1983 by short-time Fourier transform. We will also display some basic properties of this function space.

Applying the frequency-uniform localization techniques, one can get an equivalent definition of modulation spaces (see [7] for details) as follows. Let $Q_{k}$ be the unit cube with the center at $k$, so $\left\{Q_{k}\right\}_{k \in \mathbb{Z}^{n}}$ constitutes a decomposition of $\mathbb{R}^{n}$. First, we construct a smooth cut-off function. Let $\rho \in \mathcal{S}\left(\mathbb{R}^{n}\right)$ and let $\rho: \mathbb{R}^{n} \rightarrow[0,1]$ be a smooth function satisfying $\rho(\xi)=1$ for $|\xi|_{\infty} \leq 1 / 2$ and $\rho(\xi)=0$ for $|\xi| \geq 1$. Let $\rho_{k}$ be a translation of $\rho$,

$$
\rho_{k}(\xi)=\rho(\xi-k), \quad k \in \mathbb{Z}^{n} .
$$

We see that $\rho_{k}(\xi)=1$ in $Q_{k}$, so $\sum_{k \in \mathbb{Z}^{n}} \rho_{k}(\xi) \geq 1$ for all $\xi \in \mathbb{R}^{n}$. Denote

$$
\sigma_{k}(\xi)=\rho_{k}(\xi)\left(\sum_{l \in \mathbb{Z}^{n}} \rho_{l}(\xi)\right)^{-1}, \quad k \in \mathbb{Z}^{n} .
$$

Then, $\left\{\sigma_{k}(\xi)\right\}_{k \in \mathbb{Z}^{n}}$ satisfies the following properties:

$$
\begin{gathered}
\left|\sigma_{k}(\xi)\right| \geq c, \quad \forall \xi \in Q_{k}, \\
\operatorname{supp}\left(\sigma_{k}\right) \subset\left\{\xi:|\xi-k|_{\infty} \leq 1\right\}, \\
\sum_{k \in \mathbb{Z}^{n}} \sigma_{k}(\xi) \equiv 1, \quad \forall \xi \in \mathbb{R}^{n}, \\
\left|D^{\alpha} \sigma_{k}(\xi)\right| \leq C_{|\alpha|}, \quad \forall \xi \in \mathbb{R}^{n}, \alpha \in\left(\mathbb{Z}^{+} \cup\{0\}\right)^{n} .
\end{gathered}
$$

In fact, $\left\{\sigma_{k}(\xi)\right\}_{k \in \mathbb{Z}^{n}}$ constitutes a smooth decomposition of $\mathbb{R}^{n}$ and $\sigma_{k}(\xi)=\sigma(\xi-k)$, in which

$$
\sigma(\xi)=\rho(\xi)\left(\sum_{l \in \mathbb{Z}^{n}} \rho_{l}(\xi)\right)^{-1}
$$

The frequency-uniform decomposition operators can be exactly defined by

$$
\square_{k}:=\mathscr{F}^{-1} \sigma_{k} \mathscr{F}
$$

for $k \in \mathbb{Z}^{n}$.

Definition 16 (modulation space). Let $s \in \mathbb{R}, 0<p, q \leq \infty$, and one defines the modulation space

$$
\begin{aligned}
M_{p, q}^{s}\left(\mathbb{R}^{n}\right)= & \left\{f \in \mathcal{S}^{\prime}:\right. \\
& \left.\|f\|_{M_{p, q}^{s}}:=\left(\sum_{k \in \mathbb{Z}^{n}}\langle k\rangle^{s q}\left\|_{k} f\right\|_{p}^{q}\right)^{1 / q}<\infty\right\} .
\end{aligned}
$$

Below, we list some basic properties for the space $M_{p, q}^{s}\left(\mathbb{R}^{n}\right)$.

Lemma 17 (embedding). Let $s_{1}, s_{2} \in \mathbb{R}$ and $0<$ $p_{1}, p_{2}, q_{1}, q_{2} \leq \infty$.

(1) If $s_{2} \leq s_{1}, p_{1} \leq p_{2}$, and $q_{1} \leq q_{2}$, then $M_{p_{1}, q_{1}}^{s_{1}} \subset M_{p_{2}, q_{2}}^{s_{2}}$.

(2) If $q_{2}<q_{1}$ and $s_{1}-s_{2}>n / q_{2}-n / q_{1}$, then $M_{p, q_{1}}^{s_{1}} \subset M_{p, q_{2}}^{s_{2}}$.

Proposition 18 (isomorphism). Let $0<p, q \leq \infty, s, \sigma \in$ $\mathbb{R}$. Then, $J_{\sigma}=(I-\Delta)^{\sigma / 2}: M_{p, q}^{s} \rightarrow M_{p, q}^{s-\sigma}$ is an isomorphic mapping.

The proof of Proposition 18 can be found in [6] for the cases $1 \leq p, q \leq \infty$ and $[7,8]$ for the cases $0<p, q<1$. We denote $J=J_{1}$.

Lemma 19 (for uniform boundedness of $G(t)$ in $M_{p, q}^{s}$, see [9]). Let $s \in \mathbb{R}, 1 \leq p \leq \infty$, and $0<q<\infty$. One has

$$
\|G(t) f\|_{M_{p, q}^{s}} \leq C(1+|t|)^{n|1 / 2-1 / p|}\|f\|_{M_{p, q}^{s}}
$$

where the constant $C$ depends only on $p, q, s$, and $n$.

One can also find these estimates in [10] and a more general estimate on $e^{i t\left(m^{2} I+|\Delta|\right)^{1 / 2}}$ with $m \neq 0, t \geq 1$ in Chen and Fan [11]. Chen and Fan also showed that the exponent $|1 / p-1 / 2|$ is the best possible in the factor $t^{|1 / p-1 / 2|}$ if $n$ equals $1[11]$.

Lemma 20 (for truncated decay estimate of $G(t)$, see Proposition 4.2 in [2]). Let $s \in \mathbb{R}, 2 \leq p<\infty, 1 / p+1 / p^{\prime}=1$, and $0<q<\infty, \theta \in[0,1]$;

$$
2 \sigma(p)=(n+2)\left(\frac{1}{2}-\frac{1}{p}\right) .
$$

One has

$$
\|G(t) f\|_{M_{p, q}^{s}} \leq C(1+|t|)^{-n \theta(1 / 2-1 / p)}\|f\|_{M_{p^{\prime}, q}^{s+\theta 2 \sigma(p)},}
$$

where the constant $C$ depends only on $p, q, s$, and $n$.

Lemma 21 (algebra property [4]). Let $m \in \mathbb{Z}^{+}, s \geq 0$. Assume that $1 / p_{1}+\cdots+1 / p_{m}=1 / p_{0}, 1 / q_{1}+\cdots+1 / q_{m}=$ $m-1+1 / q_{0}$ with $0<p_{i} \leq \infty, 1 \leq q_{i} \leq \infty$ for $1 \leq i \leq m$. One has

$$
\left\|\prod_{i=1}^{m} u_{i}\right\|_{M_{p_{0}, q_{0}}^{s}} \leq C \prod_{i=1}^{m}\left\|u_{i}\right\|_{M_{p_{i}, q_{i}}^{s}},
$$

where $C$ is independent of $u_{i}$.

Lemma 22 (Leibniz rule for modulation space [3]). Let $m \in$ $\mathbb{Z}^{+}, s \geq 0$ and $1 / p_{1}+\cdots+1 / p_{m}=1 / p_{0}, 1 / q_{1}+\cdots+1 / q_{m}=$ $m-1+1 / q_{0}$, and $0<p_{i} \leq \infty, 1 \leq q_{i} \leq \infty$ for $1 \leq i \leq m$. One has

$$
\left\|\prod_{i=1}^{m} u_{i}\right\|_{M_{p_{0}, q_{0}}^{s}} \leq C \sum_{j=1}^{m} \prod_{i=1}^{m}\left\|u_{i}\right\|_{M_{p_{i}, q_{i}}^{\delta(i, j) s}}
$$


where $C$ is independent of $u_{i}$, and $\delta(i, j)=1$ if $i=j$ and vanishes otherwise. Particularly, if we choose $u_{i}$ to be $u$ or $\bar{u}$, $p_{i}=\infty$ and $q_{i}=1$ for $i=0,1, \ldots, m$. We have

$$
\left\|\pi\left(u^{k+1}\right)\right\|_{M_{\infty, 1}^{s}} \leq C\|u\|_{M_{\infty, 1}^{s}}\|u\|_{M_{\infty, 1}^{0}}^{k} .
$$

Lemma 23 (for Bernstein multiplier theorem, see Proposition 1.11 in [10]). Let $L \in \mathbb{Z}, L>n / 2, \partial_{x_{i}}^{\alpha} \rho \in L^{2}, i=$ $1,2, \ldots, n, 0 \leq \alpha \leq L$. Then, $\rho$ is a multiplier on $L_{p}, 1 \leq$ $p \leq \infty$. Moreover, there exists a constant $C$ such that

$$
\|\rho\|_{M_{p}} \leq C\|\rho\|_{L^{2}}^{1-n / 2 L}\left(\sum_{i=1}^{n}\left\|\partial_{x_{i}}^{L} \rho\right\|_{L^{2}}\right)^{n / 2 L}
$$

Lemma 24. Let $s \in \mathbb{R}, 0<p, q<\infty$, and let $\Omega$ be a compact subset of $\mathbb{R}^{n}$. Then, $\mathcal{S}^{\Omega}=\{f: f \in \mathcal{S}$ and Supp $\widehat{f} \subset \Omega\}$ is dense in $M_{p, q}^{s}$.

Definition 25 ( $k$-admissible pair). One calls the exponent $(p, r) k$-admissible if there exists another exponent $\gamma$ such that

$$
\begin{gathered}
\frac{1}{\gamma}+\frac{k}{r}=1, \\
\frac{1}{k+1} \leq \frac{1}{\gamma} \leq \frac{n}{n+2} \wedge n\left(\frac{1}{2}-\frac{1}{p}\right), \\
\frac{1}{k+2} \leq \frac{1}{p}<\frac{1}{2}-\frac{1}{n(k+1)} .
\end{gathered}
$$

Remark 26. From Definition 25, if $(p, r)$ is $k$-admissible, we can easily verify that

$$
k+1 \leq r<\infty, \quad r n\left(\frac{1}{2}-\frac{1}{p}\right)>1 .
$$

Moreover, we have the following inequality:

$$
\begin{aligned}
& \left\|G\left(t-t_{0}\right) u_{0}\right\|_{L_{t}^{r}\left(\mathbb{R}, M_{p, 1}^{s}\right)} \\
& \quad \leqslant\left\|\left(1+\left|t-t_{0}\right|\right)^{-n(1 / 2-1 / p)}\right\| u_{0}\left\|_{M_{p^{\prime}, 1}^{s}}\right\|_{L_{t}^{r}(\mathbb{R})} \\
& \quad \leqslant\left\|u_{0}\right\|_{M_{p^{\prime}, 1}^{s}} .
\end{aligned}
$$

Remark 27. If $1 /(k+2)+1 / n(k+1)<1 / 2$, there exist $k$ admissible pairs. The condition $1 /(k+2)+1 / n(k+1)<1 / 2$ can ensure that $2<k n$.

Definition 28. One defines the strong $M_{p, 1}^{s}$ solution to (1) as follows: the distribution $u \in C\left(I, M_{p, 1}^{s}\right) \cap C^{1}\left(I, M_{p, 1}^{s-1}\right)$ is the solution to (1) in the sense of (45) with the initial data $\left(u\left(t_{0}\right), u_{t}\left(t_{0}\right)\right)=\left(u_{0}, u_{1}\right) \in M_{p, 1}^{s} \times M_{p, 1}^{s-1}$.

We establish the following nonlinear estimate.
Proposition 29 (nonlinear estimate). Let $t_{0} \in I, F(u)=$ $\prod_{i=1}^{k+1} u_{i}$. For any $k$-admissible pair $(p, r)$, one has

$$
\begin{gathered}
\left\|\int_{t_{0}}^{t} K(t-\tau) F(u(\tau)) d \tau\right\|_{L_{t}^{r}\left(I, M_{p, 1}^{s}\right)} \\
\quad \lesssim\|F(u)\|_{L_{t}^{r /(k+1)}\left(I, M_{p, 1}^{s}\right)} \\
\quad \lesssim \prod_{i=1}^{k+1}\left\|u_{i}\right\|_{L_{t}^{r}\left(I, M_{p, 1}^{s}\right)} .
\end{gathered}
$$

Proof. Observe that $K(t) \omega^{1 / 2}=(G(t)-G(-t)) / 2 i$. For any $k$-admissible pair $(p, r)$, using the general Minkowski inequality, Proposition 18, and Lemma 20, we have

$$
\begin{aligned}
& \left\|\int_{t_{0}}^{t} K(t-\tau) F(u(\tau)) d \tau\right\|_{L_{t}^{r}\left(I, M_{p, 1}^{s}\right)} \\
& \leq\left\|\int_{t_{0}}^{t}\right\| K(t-\tau) F(u(\tau))\left\|_{M_{p, 1}^{s}} d \tau\right\|_{L_{t}^{r}(I)} \\
& \leq\left\|\int_{t_{0}}^{t}(1+|t-\tau|)^{-n \theta(1 / 2-1 / p)}\right\| F(u)\left\|_{M_{p^{\prime}, 1}^{s+\theta 2 \sigma(p)-1}} d \tau\right\|_{L_{t}^{r}(\mathbb{R})} \\
& \lesssim\left\|\int_{\mathbb{R}}(1+|t-\tau|)^{-n \theta(1 / 2-1 / p)}\right\| F(u)\left\|_{M_{p^{\prime}, 1}^{s+\theta 2 \sigma(p)-1}} \chi_{I}(\tau) d \tau\right\|_{L_{t}^{r}(\mathbb{R})}
\end{aligned}
$$

for any $\theta \in[0,1]$.

If $1 / \gamma=n /(n+2) \wedge n(1 / 2-1 / p)$, then $1 / \gamma<1$ and there exists $\theta \in(0,1]$ such that

$$
\frac{1}{\gamma}=\theta n\left(\frac{1}{2}-\frac{1}{p}\right)=\frac{n}{n+2} \wedge n\left(\frac{1}{2}-\frac{1}{p}\right) .
$$

With this $\theta$, we have $\theta 2 \sigma(p)-1 \leq 0$. Observing Definition 25 and Remark 27, we have

$$
\frac{1}{r}=\frac{k+1}{r}-\frac{1-n \theta(1 / 2-1 / p)}{1}
$$

and $r /(k+1)>1$. So in this case we choose $\theta$ satisfying (66) and exploit Lemma 17 and the Hardy-Littlewood-Sobolev inequality to have

$$
\begin{gathered}
\left\|\int_{t_{0}}^{t} K(t-\tau) F(u(\tau)) d \tau\right\|_{L_{t}^{r}\left(I, M_{p, 1}^{s}\right)} \\
\lesssim \| \int_{\mathbb{R}}(1+|t-\tau|)^{-n \theta(1 / 2-1 / p)} \\
\times\|F(u)\|_{M_{p^{\prime}, 1}^{s}} \chi_{I}(\tau) d \tau \|_{L_{t}^{r}(\mathbb{R})} \\
=\|\left(\|F(u)\|_{M_{p^{\prime}, 1}^{s}} \chi_{I}(t)\right)
\end{gathered}
$$




$$
\begin{aligned}
& *(1+|t|)^{-n \theta(1 / 2-1 / p)} \|_{\|_{L_{t}^{r}(\mathbb{R})}} \\
\lesssim & \|F(u)\|_{M_{p^{\prime}, 1}^{s}} \chi_{I}(\tau) \|_{L_{t}^{r /(k+1)}} \\
= & \|F(u)\|_{L_{t}^{r /(k+1)}\left(I, M_{p^{\prime}, 1}^{s}\right)} .
\end{aligned}
$$

If $(1 / \gamma)<n /(n+2) \wedge n(1 / 2-1 / p)$, then there exists $\theta \epsilon$ $[0,1]$ such that

$$
\frac{1}{\gamma}<\theta n\left(\frac{1}{2}-\frac{1}{p}\right) \leq \frac{n}{n+2} \wedge n\left(\frac{1}{2}-\frac{1}{p}\right) .
$$

With this $\theta$, we have $\gamma \theta n(1 / 2-1 / p)>1$ and $\theta 2 \sigma(p)-$ $1 \leq 0$. Taking advantage of Young's inequality and Hölder's inequality, we obtain

$$
\begin{gathered}
\left\|\int_{t_{0}}^{t} K(t-\tau) F(u(\tau)) d \tau\right\|_{L_{t}^{r}\left(I, M_{p, 1}^{s}\right)} \\
\lesssim\left\|(1+|t|)^{-n \theta(1 / 2-1 / p)}\right\|_{L_{t}^{\gamma}} \\
\quad \times\|\| F(u)\left\|_{M_{p^{\prime}, 1}^{s}} \chi_{I}(\tau)\right\|_{L_{t}^{r /(k+1)}} \\
\lesssim\|F(u)\|_{L_{t}^{r /(k+1)}\left(I, M_{p^{\prime}, 1}^{s}\right)} \cdot
\end{gathered}
$$

In general, we get the first result. Now, by Lemma 21 and Hölder's inequality, we have

$$
\begin{aligned}
& \left\|\int_{t_{0}}^{t} K(t-\tau) F(u(\tau)) d \tau\right\|_{L_{t}^{r}\left(I, M_{p, 1}^{s}\right)} \\
& \lesssim\|F(u)\|_{L_{t}^{r /(k+1)}\left(I, M_{p^{\prime}, 1}^{s}\right)} \\
& \lesssim\left(\int_{I}\left\|\prod_{i=1}^{k+1} u_{i}(\tau)\right\|_{M_{p l, 1}^{s}}^{r /(k+1)} d \tau\right)^{(k+1) / r} \\
& \lesssim\left(\int_{I} \prod_{i=1}^{k+1}\left\|u_{i}(\tau)\right\|_{M_{(k+1) p^{\prime}, 1}^{s}}^{r /(k+1)} d \tau\right)^{(k+1) / r} \\
& \lesssim \prod_{i=1}^{k+1}\left\|u_{i}(t)\right\|_{L_{t}^{r}\left(I, M_{(k+1) p^{\prime}, 1}^{s}\right)} .
\end{aligned}
$$

By the fact $p \leq k+2$ and the embedding theorem (Lemma 17), we obtain

$$
\prod_{i=1}^{k+1}\left\|u_{i}(t)\right\|_{L_{t}^{r}\left(I, M_{(k+1) p^{\prime}, 1}^{s}\right)} \lesssim \prod_{i=1}^{k+1}\left\|u_{i}(t)\right\|_{L_{t}^{r}\left(I, M_{p, 1}^{s}\right)} .
$$

From Proposition 29, we have an immediate corollary as follows.
Corollary 30. Let $k \in \mathbb{N}, f(u)=\pi\left(u^{k+1}\right)$, and then one has

$$
\begin{gathered}
\left\|\int_{t_{0}}^{t} K(t-\tau) f(u(\tau)) d \tau\right\|_{L_{t}^{r}\left(I, M_{p, 1}^{s}\right)} \\
\quad \leqslant\|f(u)\|_{L_{t}^{r /(k+1)}\left(I, M_{p, 1}^{s}\right)} \\
\quad \leqslant u \|_{L_{t}^{r}\left(I, M_{p, 1}^{s}\right)}^{k+1} .
\end{gathered}
$$

\section{Local Well-Posedness}

In this section, we establish the local theory for the Cauchy problem (1). In the rest of this paper, we assume that $k, n$ satisfy $1 /(k+2)+1 / n(k+1)<1 / 2$, so there exist $k$-admissible exponents by Remark 27 .

3.1. Proof of Existence Part of Theorem 1. We use the fixed point argument to construct a local solution. Let $B_{a}=\{u$ : $\left.\|u\|_{L_{t}^{r}\left(I, M_{p, 1}^{s}\right)} \leq a\right\}$, and define a map $\mathscr{J}$ on $B_{a}$ :

$$
\begin{gathered}
\mathscr{J}(v)=K^{\prime}\left(t-t_{0}\right) u_{0}+K\left(t-t_{0}\right) u_{1} \\
-\int_{t_{0}}^{t} K(t-\tau) f(v) d \tau .
\end{gathered}
$$

We want to choose suitable $a$ and $\eta$ so that $\mathscr{J}: B_{a} \rightarrow B_{a}$ is a contraction. By corollary of Proposition 29, we have

$$
\|\mathscr{J}(v)\|_{L_{t}^{r}\left(I, M_{p, 1}^{s}\right)} \leq \eta+C a^{k+1} .
$$

Let $\eta_{1}$ satisfy $C\left(2 \eta_{1}\right)^{k}=1 / 2$ and choose $\eta \leq \eta_{1}$, and then we have $\mathcal{J}: B_{2 \eta} \rightarrow B_{2 \eta}$. Indeed,

$$
\begin{aligned}
\|\mathscr{J}(v)\|_{L_{t}^{r}\left(I, M_{p, 1}^{s}\right)} & \leq \eta+C(2 \eta)^{k+1} \\
& \leq \eta+C\left(2 \eta_{1}\right)^{k}(2 \eta) \leq 2 \eta .
\end{aligned}
$$

We also have

$$
\begin{aligned}
\| \mathscr{J}(u) & -\mathscr{J}(v) \|_{L_{t}^{r}\left(I, M_{p, 1}^{s}\right)} \\
\leq & C\|f(u)-f(v)\|_{L_{t}^{r /(k+1)}\left(I, M_{p^{\prime}, 1}^{s}\right)} \\
\leq & \left.C(k+1) 2 \eta^{k}\|u-v\|_{L_{t}^{r}\left(I, M_{p, 1}^{s}\right.}\right),
\end{aligned}
$$

if $\|u\|_{L_{t}^{r}\left(I, M_{p, 1}^{s}\right)},\|v\|_{L_{t}^{r}\left(I, M_{p, 1}^{s}\right)} \in B_{2 \eta}$.

So we can shrink $\eta_{1}$ to $\eta_{2}$ so that $C(k+1) 2 \eta_{2}^{k} \leq 1 / 2$ and find a possible smaller constant $\eta_{0} \leq \eta_{2}$. Then, when $\left\|e^{i\left(t-t_{0}\right) \Delta} u_{0}\right\|_{L_{t}^{r}\left(I, M_{p, 1}^{s}\right)} \leq \eta \leq \eta_{0}$, we have $\mathcal{J}: B_{2 \eta} \rightarrow B_{2 \eta}$ and it is nothing but a contraction map. We now obtain $u \in B_{2 \eta}$ which is the fixed point of $\mathscr{J}$ that solves Cauchy problem (1) in the sense of integral form. Of course, $\|u\|_{L_{t}^{r}\left(I, M_{p, 1}^{s}\right)} \leq 2 \eta$.

3.2. Proof of Strong Solution Part of Theorem 1. In this subsection, we will verify that the local solution $u \in L_{t}^{r}\left(I, M_{p, 1}^{s}\right)$ is a $M_{p, 1}^{s}$ strong solution in the sense that $u \in C\left(I, M_{p, 1}^{s}\right) \cap$ $C^{1}\left(I, M_{p, 1}^{s-1}\right)$. 
3.2.1. To Prove $u \in C\left(I, M_{p, 1}^{s}\right)$. It is equivalent to prove that

$$
\left\|u\left(t_{n}, \cdot\right)-u(t, \cdot)\right\|_{M_{p, 1}^{s}} \longrightarrow 0
$$

as $t_{n} \rightarrow t$. We may assume without loss of generality that $t_{0}=0$,

$$
\begin{aligned}
\left\|u\left(t_{n}, \cdot\right)-u(t, \cdot)\right\|_{M_{p, 1}^{s}} & \\
\leq & \left\|K^{\prime}\left(t_{n}\right) u_{0}-K^{\prime}(t) u_{0}\right\|_{M_{p, 1}^{s}} \\
& +\left\|K\left(t_{n}\right) u_{1}-K(t) u_{1}\right\|_{M_{p, 1}^{s}} \\
& +\| \int_{0}^{t_{n}} K\left(t_{n}-\tau\right) f(u(\tau)) d \tau \\
& \quad-\int_{0}^{t} K(t-\tau) f(u(\tau)) d \tau \|_{M_{p, 1}^{s}} \\
\triangleq & I I+I I I .
\end{aligned}
$$

Recall that $u_{0}, J^{-1} u_{1} \in M_{p, 1}^{s}$. For $I, I I$, by density Lemma 24 , Lemma 19, triangle inequality, and the definition of $G(t)$, we only need to prove that $G(t) v \in C\left(I, M_{p, 1}^{s}\right)$ for $v \in \delta^{\Omega}$.

Using Hausdorff-Young inequality, we have

$$
\begin{aligned}
& \left\|\square_{k}\left(G\left(t_{n}\right) v-G(t) v\right)\right\|_{p} \\
& \quad \leq\left\|\sigma_{k}\left(e^{i t_{n}\left(1+|\xi|^{2}\right)^{1 / 2}}-e^{i t\left(1+|\xi|^{2}\right)^{1 / 2}}\right) \widehat{v}(\xi)\right\|_{p^{\prime}} \\
& \quad \leq\left\|\left(e^{i t_{n}\left(1+|\xi|^{2}\right)^{1 / 2}}-e^{i t\left(1+|\xi|^{2}\right)^{1 / 2}}\right) \widehat{v}(\xi)\right\|_{p} .
\end{aligned}
$$

Since $\widehat{v} \in \mathcal{S}$, so $\left\|\left(e^{i t_{n}\left(1+|\xi|^{2}\right)^{1 / 2}}-e^{i t\left(1+|\xi|^{2}\right)^{1 / 2}}\right) \widehat{v}(\xi)\right\|_{p} \rightarrow 0$ as $t_{n} \rightarrow t$, by Lebesgue's dominated convergence theorem. Since $v \in \mathcal{S}^{\Omega}$, there exists only the finite number of $k$ such that $\square_{k}\left(G\left(t_{n}\right) v-G(t) v\right) \neq 0$, so we have

$$
\left\|G\left(t_{n}\right) v-G(t) v\right\|_{M_{p, 1}^{s}} \longrightarrow 0 \quad \text { as } t_{n} \longrightarrow t .
$$

Thus,

$$
\begin{gathered}
I=\left\|K^{\prime}\left(t_{n}\right) u_{0}-K^{\prime}(t) u_{0}\right\|_{M_{p, 1}^{s}} \longrightarrow 0 \quad \text { as } t_{n} \longrightarrow t, \\
I I=\left\|K\left(t_{n}\right) u_{1}-K(t) u_{1}\right\|_{M_{p, 1}^{s}} \longrightarrow 0 \quad \text { as } t_{n} \longrightarrow t .
\end{gathered}
$$

For III,

$$
\begin{aligned}
I I I= & \| \int_{0}^{t_{n}} K\left(t_{n}-\tau\right) f(u(\tau)) d \tau \\
& -\int_{0}^{t} K(t-\tau) f(u(\tau)) d \tau \|_{M_{p, 1}^{s}} \\
\leq & \| \int_{0}^{t_{n}} K\left(t_{n}-\tau\right) f(u(\tau)) d \tau \\
& \quad-\int_{0}^{t_{n}} K(t-\tau) f(u(\tau)) d \tau \|_{M_{p, 1}^{s}}
\end{aligned}
$$

$$
\begin{aligned}
& +\| \int_{0}^{t_{n}} K(t-\tau) f(u(\tau)) d \tau \\
& \quad-\int_{0}^{t} K(t-\tau) f(u(\tau)) d \tau \|_{M_{p, 1}^{s}} \\
& \triangleq \widetilde{I}+\widetilde{I I} .
\end{aligned}
$$

First, by the Minkowski inequality, we have that

$$
\begin{aligned}
\widetilde{I} & =\left\|\int_{0}^{t_{n}}\left(K\left(t_{n}-\tau\right)-K(t-\tau)\right) f(u(\tau)) d \tau\right\|_{M_{p, 1}^{s}} \\
& \leq \int_{0}^{t_{n}}\left\|\left(K\left(t_{n}-\tau\right)-K(t-\tau)\right) f(u(\tau))\right\|_{M_{p, 1}^{s}} d \tau .
\end{aligned}
$$

For $\left\|\left(K\left(t_{n}-\tau\right)-K(t-\tau)\right) f(u(\tau))\right\|_{M_{p, 1}^{s}} \lesssim\|f(u(\tau))\|_{M_{p^{\prime}, 1}^{s}} \lesssim$ $\|u\|_{M_{p, 1}^{s}}^{k+1}$ and $k+1 \leq r$, we have $\|u\|_{M_{p, 1}^{s}}^{k+1} \in L^{1}$, so $\left\|\left(K\left(t_{n}-\tau\right)-K(t-\tau)\right) f(u(\tau))\right\|_{M_{p, 1}^{s}} \in L^{1}$. Since $\left\|\left(K\left(t_{n}-\tau\right)-K(t-\tau)\right) f(u(\tau))\right\|_{M_{p, 1}^{s}} \stackrel{\rightarrow}{\rightarrow} 0$, as $t_{n} \rightarrow t$, therefore, $\widetilde{I} \rightarrow 0$, as $t_{n} \rightarrow t$.

Secondly, as in the proof of Proposition 29, we get that

$$
\begin{gathered}
\widetilde{I I} \lesssim \int_{t_{n}}^{t}(1+|t-\tau|)^{-n(1 / 2-1 / p)} \\
\times\|f(u(\tau))\|_{M_{p^{\prime}, 1}^{s}} d \tau \\
\lesssim \int_{t_{n}}^{t}\|f(u(\tau))\|_{M_{p^{\prime}, 1}^{s}} d \tau .
\end{gathered}
$$

Since $\|f(u)\|_{M_{p^{\prime}, 1}^{s}} \leqslant\|u\|_{M_{p, 1}^{s}}^{k+1},\|u\|_{M_{p, 1}^{s}}^{k+1} \in L^{1}$. We have

$$
\widetilde{I I} \longrightarrow 0, \quad \text { as } t_{n} \longrightarrow t
$$

Because $I I I \leq \widetilde{I}+\widetilde{I I}$, it leads to

$$
I I I \longrightarrow 0, \quad \text { as } t_{n} \longrightarrow t .
$$

Accordingly, (78) holds; that is, $u \in C\left(I, M_{p, 1}^{s}\right)$.

3.2.2. $u_{t}(t)$ Exists and Is Continuous in $M_{p, 1}^{s-1}$ Sense. First of all, we consider the existence of derivative in $M_{p, 1}^{s-1}$ sense.

Recall that

$$
\begin{array}{r}
u(t)=K^{\prime}\left(t-t_{0}\right) u_{0}+K\left(t-t_{0}\right) u_{1} \\
-\int_{t_{0}}^{t} K(t-\tau) f(u(\tau)) d \tau
\end{array}
$$

For $u_{0}, J^{-1} u_{1} \in M_{p, 1}^{s}$, and the definition of $G(t)$, we should only deal with the derivatives of $G(t) \psi(x)$ for $\psi \in M_{p, 1}^{s}$ and $\int_{t_{0}}^{t} K(t-\tau) f(u(\tau)) d \tau$. 
By the density Lemma 24 , for every $\varepsilon>0$, there exists $v \in \mathcal{S}^{\Omega} \cap M_{p, 1}^{s}$, such that $\|\psi-v\|_{M_{p, 1}^{s}}<\varepsilon$. For the derivative of $G(t) \psi(x)$ at $t=t_{3}$ for $\psi \in M_{p, 1}^{s}$, we have

$$
\begin{aligned}
& \left\|\frac{G(t) \psi-G\left(t_{3}\right) \psi}{t-t_{3}}-i \omega^{1 / 2} G\left(t_{3}\right) \psi\right\|_{M_{p, 1}^{s-1}} \\
& =\left\|\frac{G(t) \psi-G\left(t_{3}\right) \psi}{\left(t-t_{3}\right) \omega^{1 / 2}}-i G\left(t_{3}\right) \psi\right\|_{M_{p, 1}^{s}} \\
& \leq\left\|\frac{G(t)(\psi-v)-G\left(t_{3}\right)(\psi-v)}{\left(t-t_{3}\right) \omega^{1 / 2}}\right\|_{M_{p, 1}^{s}} \\
& \quad+\left\|\frac{G(t) v-G\left(t_{3}\right) v}{\left(t-t_{3}\right) \omega^{1 / 2}}-i G\left(t_{3}\right) v\right\|_{M_{p, 1}^{s}} \\
& \quad+\left\|i G\left(t_{3}\right)(\psi-v)\right\|_{M_{p, 1}^{s-1}} \\
& \triangleq I V+V+V I .
\end{aligned}
$$

For $V$, by the Hausdorff-Young inequality and the Lebesgue dominated convergence theorem, we have

$$
\begin{aligned}
& \left\|\square_{k}\left(\frac{G(t) v-G\left(t_{3}\right) v}{\left(t-t_{3}\right) \omega^{1 / 2}}-i G\left(t_{3}\right) v\right)\right\|_{p} \\
& \quad \leq\left\|\sigma_{k}\left(\frac{e^{i t\langle\xi\rangle}-e^{i t_{3}\langle\xi\rangle}}{\left(t-t_{3}\right)\langle\xi\rangle}-i e^{i t_{3}\langle\xi\rangle}\right) \widehat{v}\right\|_{p^{\prime}} \\
& \quad \longrightarrow 0 \quad \text { as } t \longrightarrow t_{3} .
\end{aligned}
$$

As $v \in \mathcal{S}^{\Omega} \cap M_{p, 1}^{s}$, so there is only the finite number of $k$ such that $\square_{k}\left(\left(G(t) v-G\left(t_{3}\right) v\right) /\left(t-t_{3}\right) \omega^{1 / 2}-i G\left(t_{3}\right) v\right) \neq 0$. Thus, we get $V \rightarrow 0$ as $t \rightarrow t_{3}$; that is, $(G(t) v(x))_{t}=i \omega^{1 / 2} G(t) v(x)$ in $M_{p, 1}^{s-1}$, for $v \in \mathcal{S}^{\Omega} \cap M_{p, 1}^{s}$.

For $I V$, by the Bernstein multiplier theorem, we have

$$
\left\|\square_{l} \frac{G(t)(\psi-v)-G\left(t_{3}\right)(\psi-v)}{\left(t-t_{3}\right) \omega^{1 / 2}}\right\|_{p} \leqslant\|\psi-v\|_{p} .
$$

Using the almost orthogonality of modulation space, we obtain $I V \lesssim \psi-v_{M_{p, 1}^{s}}<\varepsilon$.

For VI, by Lemma 19, we have $V I=\left\|i G\left(t_{3}\right)(\psi-v)\right\|_{M_{p, 1}^{s}} \leqslant$ $\|\psi-v\|_{M_{p, 1}^{s}}<\varepsilon$.

Accordingly, for $\psi \in M_{p, 1}^{s}$,

$$
(G(t) \psi)_{t}=i \omega^{1 / 2} G(t) \psi \quad \text { in } M_{p, 1}^{s-1} .
$$

For the nonlinear part,

$$
\begin{aligned}
& \| \frac{\int_{t_{0}}^{t} K(t-\tau) f(u(\tau)) d \tau-\int_{t_{0}}^{t_{3}} K\left(t_{3}-\tau\right) f(u(\tau)) d \tau}{t-t_{3}} \\
& -\int_{t_{0}}^{t_{3}} K^{\prime}\left(t_{3}-\tau\right) f(u(\tau)) d \tau \|_{M_{p, 1}^{s-1}} \\
& \leq \| \frac{\int_{t_{0}}^{t_{3}}\left(K(t-\tau)-K\left(t_{3}-\tau\right)\right) f(u(\tau)) d \tau}{t-t_{3}} \\
& -\int_{t_{0}}^{t_{3}} K^{\prime}\left(t_{3}-\tau\right) f(u(\tau)) d \tau \|_{M_{p, 1}^{s-1}} \\
& +\left\|\frac{\int_{t_{3}}^{t} K(t-\tau) f(u(\tau)) d \tau}{t-t_{3}}\right\|_{M_{p, 1}^{s s-1}} \\
& =\| \int_{t_{0}}^{t_{3}}\left(\frac{\left(K(t-\tau)-K\left(t_{3}-\tau\right)\right)}{t-t_{3}}-K^{\prime}\left(t_{3}-\tau\right)\right) \\
& \times f(u(\tau)) d \tau \|_{M_{p, 1}^{s-1}} \\
& +\left\|\frac{\int_{t_{3}}^{t} K(t-\tau) f(u(\tau)) d \tau}{t-t_{3}}\right\|_{M_{p, 1}^{s-1}} \\
& \lesssim \int_{t_{0}}^{t_{3}} \|\left(\frac{\left(K(t-\tau)-K\left(t_{3}-\tau\right)\right)}{t-t_{3}}-K^{\prime}\left(t_{3}-\tau\right)\right) \\
& \times f(u(\tau)) \|_{M_{p, 1}^{s-1}} d \tau \\
& +\max _{\tau \in\left[t, t_{3}\right] \operatorname{or}\left[t_{3}, t\right]}\|K(t-\tau) f(u(\tau))\|_{M_{p, 1}^{s-1}} .
\end{aligned}
$$

If $\omega(t, x) \in C\left(I, M_{p, 1}^{s}\right)$, then we have $K(t) \omega(t, x) \in$ $C\left(I, M_{p, 1}^{s-1}\right)$. In fact, taking advantage of (92) and the Lebesgue dominated convergence theorem, we can get

$$
\begin{aligned}
& \left\|K(t) \omega(t, x)-K\left(t_{3}\right) \omega\left(t_{3}, x\right)\right\|_{M_{p, 1}^{s-1}} \\
& \leq\left\|\left(K(t)-K\left(t_{3}\right)\right) \omega\left(t_{3}, x\right)\right\|_{M_{p, 1}^{s-1}} \\
& \quad+\left\|K(t)\left(\omega(t, x)-\omega\left(t_{3}, x\right)\right)\right\|_{M_{p, 1}^{s-1}} \\
& \quad \longrightarrow 0 \quad \text { as } t \longrightarrow t_{3} .
\end{aligned}
$$

Recall that $f(u) \in C\left(I, M_{p, 1}^{s}\right)$ and apply (92) and the Lebesgue dominated convergence theorem to the first term of (93); we have

$$
\begin{aligned}
&\left.\left(\int_{t_{0}}^{t} K(t-\tau) f(u(\tau)) d \tau\right)_{t}\right|_{t=t_{3}} \\
& \quad=\int_{t_{0}}^{t_{3}} K^{\prime}\left(t_{3}-\tau\right) f(u(\tau)) d \tau \quad \text { in } M_{p, 1}^{s-1} .
\end{aligned}
$$


Consequently,

$$
\begin{aligned}
u_{t}(t)= & -J^{2} K\left(t-t_{0}\right) u_{0}+K^{\prime}\left(t-t_{0}\right) u_{1} \\
& -\int_{t_{0}}^{t} K^{\prime}(t-\tau) f(u(\tau)) d \tau \text { in } M_{p, 1}^{s-1} .
\end{aligned}
$$

Next, the proof of time continuity of $u_{t}$ is similar to $u$. It only needs to take care of the difference of smoothness and the action of the Bessel potential. Finally, we obtain $u \in$ $C\left(I, M_{p, 1}^{s}\right) \cap C^{1}\left(I, M_{p, 1}^{s-1}\right)$; that is, it is the strong $M_{p, 1}^{s}$ solution.

3.3. Global Well-Posedness for Small Fine Data. Let us construct a time decaying norm as

$$
\Gamma(u)=\sup _{t \in \mathbb{R}}(1+|t|)^{n(1 / 2-1 / p)}\|u\|_{M_{p, 1}^{s}} .
$$

This idea for the NLS can be traced back to the work of Strauss [12] and Wang and Hudzik [2]. We consider the following mapping:

$$
\begin{aligned}
\mathscr{T}: u(t) \hookrightarrow & K^{\prime}(t) u_{0}+K(t) u_{1} \\
& -\int_{0}^{t} K(t-\tau) f(u(\tau)) d \tau
\end{aligned}
$$

in the metric space $\mathscr{D}=\{u: \Gamma(u) \leq M\}$ with $d(u, v)=\Gamma(u-$ $v$ ). For any $u \in \mathscr{D}$, in view of Lemma 20 , we get that

$$
\begin{aligned}
\Gamma(\mathscr{T} u) \leqslant & \left\|u_{0}\right\|_{M_{p^{\prime}, 1}^{s+2 \sigma(p)}}+\left\|u_{1}\right\|_{M_{p^{\prime}, 1}^{s+2 \sigma(p)-1}} \\
& +\Gamma\left(\int_{0}^{t} K(t-\tau) f(u(\tau)) d \tau\right)
\end{aligned}
$$

and (recall the notation that $\langle t\rangle=1+|t|)$

$$
\begin{aligned}
\Gamma\left(\int_{0}^{t} K(t-\tau) f(u(\tau)) d \tau\right) \\
\lesssim \sup _{t \in \mathbb{R}}\langle t\rangle^{n(1 / 2-1 / p)} \\
\quad \times \int_{0}^{t}\langle t-\tau\rangle^{-n(1 / 2-1 / p)} \\
\quad \times\|u\|_{M_{p, 1}^{s}} d \tau \\
\lesssim(\Gamma(u))^{k+1} \sup _{t \in \mathbb{R}}\langle t\rangle^{n(1 / 2-1 / p)} \\
\quad \times \int_{0}^{t}\langle t-\tau\rangle^{-n(1 / 2-1 / p)} \\
\quad \times\langle\tau\rangle^{-n(1 / 2-1 / p)(k+1)} d \tau .
\end{aligned}
$$

Owing to $n(1 / 2-1 / p)(k+1)>1$, it follows that

$$
\begin{aligned}
\int_{0}^{t / 2} & \langle t-\tau\rangle^{-n(1 / 2-1 / p)}\langle\tau\rangle^{-n(1 / 2-1 / p)(k+1)} d \tau \\
& \leqslant\langle t\rangle^{-n(1 / 2-1 / p)} \int_{0}^{t / 2}\langle\tau\rangle^{-n(1 / 2-1 / p)(k+1)} d \tau \\
& \leqslant\langle t\rangle^{-n(1 / 2-1 / p)}
\end{aligned}
$$

and that

$$
\begin{aligned}
\int_{t / 2}^{t}\langle t & -\tau\rangle^{-n(1 / 2-1 / p)}\langle\tau\rangle^{-n(1 / 2-1 / p)(k+1)} d \tau \\
& =\int_{0}^{t / 2}\langle\tau\rangle^{-n(1 / 2-1 / p)}\langle t-\tau\rangle^{-n(1 / 2-1 / p)(k+1)} d \tau \\
& \leq\langle t\rangle^{-n(1 / 2-1 / p)}\langle t\rangle^{-k n(1 / 2-1 / p)} \int_{0}^{t / 2}\langle\tau\rangle^{-(1 / 2-1 / p)} d \tau \\
& \leq\langle t\rangle^{-n(1 / 2-1 / p)}
\end{aligned}
$$

Combining the above four inequalities, we have

$$
\Gamma(\mathscr{T} u) \leqslant\left\|u_{0}\right\|_{M_{p^{\prime}, 1}^{s+2 \sigma(p)}}+\left\|u_{1}\right\|_{M_{p^{\prime}, 1}^{s+2 \sigma(p)-1}}+\Gamma(u)^{k+1} .
$$

Hence, if we choose $\delta \leqslant M / 2$ and $M$ small enough, then using the standard contraction mapping argument the Cauchy problem (1) has a unique solution satisfying (13).

3.4. Uniqueness. In order to prove the uniqueness in a category of strong solution, we only need to verify it locally; that is, to prove that the set $\{t \in I: u=v\}$ is open. If $u\left(t_{1}\right)=v\left(t_{1}\right)$ for $t_{1} \in I$, we can choose an interval $I_{1}$ sufficient small so that $t_{1} \in I_{1} \subset I$ and

$$
\begin{aligned}
& \|u-v\|_{L_{t}^{\infty}\left(I_{1}, M_{p, 1}^{s}\right)} \\
& \leq C\left(\left|I_{1}\right|, k\right) \max \left\{\|u\|_{L_{t}^{\infty}\left(I_{1}, M_{p, 1}^{s}\right)},\right. \\
& \left.\|v\|_{L_{t}^{\infty}\left(I_{1}, M_{p, 1}^{s}\right)}\right\} \\
& \quad \times\|u-v\|_{L_{t}^{\infty}\left(I_{1}, M_{p, 1}^{s}\right)} \\
& \leq \frac{1}{2}\|u-v\|_{L_{t}^{\infty}\left(I_{1}, M_{p, 1}^{s}\right)} .
\end{aligned}
$$

So $u=v$ on $I_{1}$, which concludes that $t_{1}$ is an interior point. So $I$ is open.

3.5. Blowup Criterion. We assume that $\|u\|_{L_{t}^{r}\left(\left[t_{0}, T\right], M_{p, 1}^{s}\right)}<\infty$, write the integral equation on $\left[t_{m}, T\right]$, and use nonlinear estimate to deduce that

$$
\begin{gathered}
\left\|K^{\prime}\left(t-t_{m}\right) u\left(t_{m}\right)+K\left(t-t_{m}\right) u_{t}\left(t_{m}\right)\right\|_{L_{t}^{r}\left(\left[t_{m}, T\right], M_{p, 1}^{s}\right)} \\
\quad \leq\|u\|_{L_{t}^{r}\left(\left[t_{m}, T\right], M_{p, 1}^{s}\right)}+\|u\|_{L_{t}^{r}\left(\left[t_{m}, T\right], M_{p, 1}^{s}\right)}^{k+1} .
\end{gathered}
$$

Then, let $t_{m}$ close to $T$ sufficiently enough so that $\left\|K^{\prime}\left(t-t_{m}\right) u\left(t_{m}\right)+K\left(t-t_{m}\right) u_{t}\left(t_{m}\right)\right\|_{L_{t}^{r}\left(\left[t_{m}, T\right], M_{p, 1}^{s}\right)}<(1 / 2) \eta_{0}$, where $\eta_{0}$ is the one we chose in Theorem 1 . Then, $\exists \delta>0$, such that $\left\|K^{\prime}\left(t-t_{m}\right) u\left(t_{m}\right)+K\left(t-t_{m}\right) u_{t}\left(t_{m}\right)\right\|_{L_{t}^{r}\left(\left[t_{m}, T+\delta\right], M_{p, 1}^{s}\right)} \leq \eta_{0}$, so we can extend $u$ on $\left[t_{0}, T+\delta\right]$ by local existence and uniqueness, a contradiction.

3.6. Persistence of Regularity. We want the norm which can hold the continuation of solution to be as low regularity 
as possible. It is interesting to make an assertion that the boundedness of low regularity norm $\|u(t)\|_{M_{p_{2}, 1}^{s_{2}}}$ suffices to ensure the boundedness of high regularity norm $\|u(t)\|_{M_{p_{1}, 1}^{s_{1}}}$ and thus ensure the continuation of strong $M_{p_{1}, 1}^{s_{1}}$ solution. By the improved version of product lemma (Lemma 22) and the embedding theorems of modulation spaces, we can establish Lemmas 31 and 32 to achieve our goal.

Firstly, we "reduce" the regularity index from $p$ to $\infty$.

Lemma 31. Let $I$ be a bounded time interval containing $t_{0}$, let $p \in[1, \infty]$, and let $u \in C\left(I, M_{p, 1}^{s}\right)$ be a strong $M_{p, 1}^{s}$ solution to (1). If the quantity $\|u\|_{L_{t}^{k}\left(I, M_{\infty}^{s}, 1\right.}$ is finite, then one has

$$
\begin{aligned}
& \|u(t)\|_{L_{t}^{\infty}\left(I, M_{p, 1}^{s}\right)} \\
& \leq C(|I|)\left(\left\|u\left(t_{0}\right)\right\|_{M_{p, 1}^{s}}+\left\|u_{t}\left(t_{0}\right)\right\|_{M_{p, 1}^{s-1}}\right) \\
& \quad \times \exp \left(\int_{I} C(|I|)\|u(\tau)\|_{M_{\infty}^{s} 1}^{k} d \tau\right),
\end{aligned}
$$

where $C(|I|)$ is some positive constant associated with the length of $I$.

Proof. Without loss of generality, we may assume $t_{0}=\inf I$ and $I=\left[t_{0}, T\right]$ for some $T>t_{0}$. Writing the integral equation, using Lemma 19, and embedding, we have

$$
\begin{aligned}
\|u(t)\|_{M_{p, 1}^{s}} \leq C(|I|)( & \left\|u\left(t_{0}\right)\right\|_{M_{p, 1}^{s}} \\
& \left.+\left\|u_{t}\left(t_{0}\right)\right\|_{M_{p, 1}^{s-1}}+\int_{t_{0}}^{t}\|f(u(\tau))\|_{M_{p, 1}^{s}} d \tau\right) \\
\leq C(|I|)( & \left\|u\left(t_{0}\right)\right\|_{M_{p, 1}^{s}}+\left\|u_{t}\left(t_{0}\right)\right\|_{M_{p, 1}^{s-1}} \\
& \left.+\int_{t_{0}}^{t}\|(u(\tau))\|_{M_{\infty, 1}^{s}}^{k}\|(u(\tau))\|_{M_{p, 1}^{s}} d \tau\right)
\end{aligned}
$$

for $t \in\left[t_{0}, T\right]$. Let $A(t)=\|u(t)\|_{M_{p, 1}^{s}}$ and $B(t)=\|(u(t))\|_{M_{\infty, 1}^{s}}^{k}$, and both $A$ and $B$ are continuous and nonnegative on $\left[t_{0}, T\right]$. Using Gronwall inequality, we have

$$
\begin{aligned}
\|u(t)\|_{M_{p, 1}^{s}} \leqslant & C(|I|)\left(\left\|u\left(t_{0}\right)\right\|_{M_{p, 1}^{s}}+\left\|u_{t}\left(t_{0}\right)\right\|_{M_{p, 1}^{s-1}}\right) \\
& \times \exp \left(\int_{t_{0}}^{t} C(|I|)\|u(\tau)\|_{M_{\infty}^{s} 1}^{k} d \tau\right)
\end{aligned}
$$

for $t \in\left[t_{0}, T\right]$ and obtain the conclusion immediately.

Secondly, we reduce another regularity index from $s$ to 0 . By the Leibniz rules for modulation space Lemma 22, we can deduce the following lemma. The proof of this lemma is very similar to the proof of Lemma 31, so we omit the details.
Lemma 32. Let $I$ be a bounded time interval containing $t_{0}$, $s \geq 0$, and let $u \in C\left(I, M_{\infty, 1}^{s}\right)$ be a strong $M_{\infty, 1}^{s}$ solution to (1). If the quantity $\|u\|_{L_{t}^{k}\left(I, M_{\infty, 1}^{0}\right)}$ is finite, then one has

$$
\begin{aligned}
& \|u(t)\|_{L_{t}^{\infty}\left(I, M_{\infty, 1}^{s}\right)} \\
& \quad \leq C(|I|)\left(\left\|u\left(t_{0}\right)\right\|_{M_{\infty, 1}^{s}}+\left\|u_{t}\left(t_{0}\right)\right\|_{M_{\infty, 1}^{s-1}}\right) \\
& \quad \times \exp \left(\int_{I} C(|I|)\|u(\tau)\|_{M_{\infty, 1}^{0}}^{k} d \tau\right),
\end{aligned}
$$

where $C(|I|)$ is some positive constant associated with the length of $I$.

So, for any strong $M_{p, 1}^{s}$ solution $u$ in a bounded interval $I$, if it keeps $L_{t}^{k}\left(I, M_{\infty, 1}^{0}\right)$ norm bounded, it will keep $M_{p, 1}^{s}$ norm bounded in the same interval. Conversely, if $\|u(t)\|_{M_{p, 1}^{s}}$ is bounded in a bounded time interval $I$, by embedding $M_{p, 1}^{s} \subset M_{\infty, 1}^{0}, u(t)$ will be in $L_{t}^{k}\left(I, M_{\infty, 1}^{0}\right)$. Thus, one can extend a strong solution in $M_{p, 1}^{s}$ if and only if the quantity $\|u\|_{L_{t}^{k}\left(I, M_{\infty, 1}^{0}\right)}$ remains bounded. This implies that the blowup phenomenon is independent of exact $p$ and $s$, so if an initial data $\left(u\left(t_{0}\right), u_{t}\left(t_{0}\right)\right)$ lies in both $M_{p_{1}, 1}^{s_{1}} \times M_{p_{1}, 1}^{s_{1}-1}$ and $M_{p_{2}, 1}^{s_{2}} \times M_{p_{2}, 1}^{s_{2}-1}$ $\left(1 \leq p_{1}, p_{2} \leq \infty, 0<s_{1}, s_{2}<\infty\right)$, they have the same maximal existence interval.

\section{Scattering Theorems}

The goal of this section is to derive scattering results.

4.1. Proof of Scattering Theorem. Without loss of generality, we assume $t_{0}=0$, and let

$$
\begin{aligned}
& 2 v_{1}(t)=u_{0}+\frac{u_{1}}{i \omega^{1 / 2}}-\int_{0}^{t} \frac{G(-\tau) f(u(\tau))}{i \omega^{1 / 2}} d \tau, \\
& 2 v_{2}(t)=u_{0}-\frac{u_{1}}{i \omega^{1 / 2}}+\int_{0}^{t} \frac{G(\tau) f(u(\tau))}{i \omega^{1 / 2}} d \tau .
\end{aligned}
$$

For $0<s<t$,

$$
v_{1}(t)-v_{1}(s)=-\int_{s}^{t} \frac{G(-\tau) f(u(\tau))}{i \omega^{1 / 2}} d \tau .
$$

By the fact we used in Section 3.2.1 that is from Definition 25 for $k$-admissible pair $(p, r)$, there exists $\tilde{\gamma} \geq 1$ such that

$$
\frac{1}{\tilde{\gamma}}+\frac{k+1}{r}=1, \quad \tilde{\gamma} n\left(\frac{1}{2}-\frac{1}{p}\right)>1 .
$$


Take advantage of the nonlinear estimate and Hölder inequality:

$$
\begin{aligned}
\| v_{1}(t)- & v_{1}(s) \|_{M_{p, 1}^{s}} \\
\lesssim & \int_{s}^{t}(1+|\tau|)^{-n(1 / 2-1 / p)} \\
& \times\|f(u(\tau))\|_{M_{p^{\prime}, 1}^{s}} d \tau \\
\lesssim & \int_{s}^{t}(1+|\tau|)^{-n(1 / 2-1 / p)} \\
& \times\|u\|_{M_{p, 1}^{s}}^{k+1} d \tau \\
\lesssim & \left\|(1+|t|)^{-n(1 / 2-1 / p)}\right\|_{L_{t}^{\tilde{\gamma}}} \\
& \times\|\| u\left\|_{M_{p, 1}^{s}}^{k+1}\right\|_{L_{t}^{r /(k+1)}([s, t])} \\
\lesssim & \left\|(1+|t|)^{-\tilde{\gamma} n(1 / 2-1 / p)}\right\|_{L_{t}^{1}}^{1 / \tilde{\gamma}} \\
& \times\|u\|_{L_{t}^{r}\left([s, t], M_{p, 1}^{s}\right)}^{k+1} \\
\lesssim & \|u\|_{L_{t}^{r}\left([s, t], M_{p, 1}^{s}\right)}^{k+1}
\end{aligned}
$$

Since $\|u\|_{L_{t}^{r}\left(\mathbb{R}, M_{p, 1}^{s}\right)} \leq M$, we have

$$
\left\|v_{1}(t)-v_{1}(s)\right\|_{M_{p, 1}^{s}} \leqslant\|u\|_{L_{t}^{r}\left([s, t], M_{p, 1}^{s}\right)}^{k+1} \longrightarrow 0
$$

as $t, s \rightarrow \infty$. This implies that $v(t)$ is Cauchy in $M_{p, 1}^{s}$ as $t \longrightarrow$ $\infty$. Denote $v_{1}^{+}$to be the limit:

$$
\begin{aligned}
2 v_{1}^{+} & =\lim _{t \rightarrow+\infty} 2 v_{1}(t) \\
& =u_{0}+\frac{u_{1}}{i \omega^{1 / 2}}-\int_{0}^{+\infty} \frac{G(-\tau) f(u(\tau))}{i \omega^{1 / 2}} d \tau, \\
2 v_{1}^{-} & =\lim _{t \rightarrow-\infty} 2 v_{1}(t) \\
& =u_{0}-\frac{u_{1}}{i \omega^{1 / 2}}+\int_{0}^{-\infty} \frac{G(\tau) f(u(\tau))}{i \omega^{1 / 2}} d \tau .
\end{aligned}
$$

In a similar way, we obtain

$$
v_{2}^{+}=\lim _{t \rightarrow+\infty} v_{2}(t), \quad v_{2}^{-}=\lim _{t \rightarrow-\infty} v_{2}(t) .
$$

Recall that $v^{+}=G(t) v_{1}^{+}+G(-t) v_{2}^{+}$; so taking advantage of the nonlinear estimates, we get

$$
\begin{aligned}
\| u(t) & -v^{+} \|_{M_{p, 1}^{s}} \\
& =\left\|\int_{t}^{+\infty} K(t-\tau) f(u(\tau)) d \tau\right\|_{M_{p, 1}^{s}}
\end{aligned}
$$

$$
\begin{aligned}
& \leq\left\|(1+|t|)^{-\widetilde{\gamma} n(1 / 2-1 / p)}\right\|_{L_{t}^{1}}^{1 / \tilde{\gamma}} \\
& \times\|u\|_{L_{t}^{r}\left([t, \infty], M_{p, 1}^{s}\right)}^{k+1} \longrightarrow 0
\end{aligned}
$$

as $t \rightarrow+\infty$. So is $v^{-}$, respectively. In fact, in our proof, we also have $v_{1}^{+} \in M_{p, 1}^{s}$.

One may ask whether there exists a global $M_{p, 1}^{s}$ solution to Cauchy problem (1) which is scattering associated with $v_{1}^{+}, v_{2}^{+} \in M_{p, 1}^{s}$. The following remark gives us a partial answer.

Remark 33. Let $v_{1}^{+}, v_{2}^{+} \in M_{p, 1}^{s}$ and $G(t) v_{1}^{+}+G(-t) v_{2}^{+} \in$ $L_{t}^{r}\left(\left[T_{1}, \infty\right), M_{p, 1}^{s}\right)$, and then there exists a solution $u$ of $(1)$ on $I=[T, \infty)$ for some $T>T_{1}$ such that

$$
\left\|u(t)-\left(G(t) v_{1}^{+}+G(-t) v_{2}^{+}\right)\right\|_{M_{p, 1}^{s}} \longrightarrow 0
$$

as $t \rightarrow+\infty$.

To construct $u$, we only need to solve the Cauchy problem from $t=+\infty$ to some finite time $t=T>T_{1}$ with initial data at $t=+\infty$, that is, to solve the following equation:

$$
\begin{aligned}
u(t)= & G(t) v_{1}^{+}+G(-t) v_{2}^{+} \\
& +\int_{t}^{+\infty} \frac{G(t-\tau)+G(\tau-t)}{2 i \omega^{1 / 2}} f(u) d \tau
\end{aligned}
$$

on $I=[T, \infty)$. In fact, we have $\left\|G(t) v_{1}^{+}+G(-t) v_{2}^{+}\right\|_{L_{t}^{r}\left([T, \infty), M_{p, 1}^{s}\right)}<\eta_{0}$. If we choose $T>T_{1}$ large enough, where $\eta_{0}$ is as in Theorem 1 , then we can solve this equation by the same technique used in the proof of Theorem 1 and deduce that there exists a solution to (1) on $I=[T, \infty)$ and $\|u(t)\|_{L_{t}^{r}\left(\left[T_{1}, \infty\right), M_{p, 1}^{s}\right)}<\infty$. So, if $t$ is large enough, we have

$$
\begin{aligned}
\| u(t) & -\left(G(t) v_{1}^{+}+G(-t) v_{2}^{+}\right) \|_{M_{p, 1}^{s}} \\
& =\left\|\int_{t}^{\infty} K(t-\tau) f(u(\tau)) d \tau\right\|_{M_{p, 1}^{s}} \\
& \leqslant\|u\|_{L_{t}^{r}\left([t, \infty], M_{p, 1}^{s}\right)}^{k+1} \longrightarrow 0
\end{aligned}
$$

as $t \rightarrow \infty$. A similar argument applies in the situation $t \rightarrow$ $-\infty$.

\section{Stability Theory}

In this section, we will derive the stability theory including short-time result and long-time result and deduce some corollaries. 
5.1. Proof of Short-Time Perturbations. By time symmetry, we may assume that $t_{0}=\inf I$. Let $v=u-\widetilde{u}$. Then, $v$ satisfies the following initial value problem:

$$
\begin{gathered}
\left(\partial_{t t}+I-\Delta\right) v=f(\widetilde{u}+v)-f(\widetilde{u})-e, \\
v\left(t_{0}, x\right)=u\left(t_{0}, x\right)-\widetilde{u}\left(t_{0}, x\right) \\
v_{t}\left(t_{0}, x\right)=u_{t}\left(t_{0}, x\right)-\widetilde{u}_{t}\left(t_{0}, x\right)
\end{gathered}
$$

The integral equation is

$$
\begin{aligned}
v(t)= & K^{\prime}\left(t-t_{0}\right) v\left(t_{0}\right)+K\left(t-t_{0}\right) v_{t}\left(t_{0}\right) \\
& -\int_{t_{0}}^{t} K(t-\tau)(f(\widetilde{u}+v)-f(\widetilde{u})-e) d \tau
\end{aligned}
$$

For $T \in I$, define

$$
S(T)=\left\|\left(\partial_{t t}+I-\Delta\right) v+e\right\|_{L_{t}^{r /(k+1)}\left(\left[t_{0}, T\right], M_{p^{\prime}, 1}^{s}\right.} .
$$

By nonlinear estimate and the smallness condition, for every $T \in I$,

$$
\begin{aligned}
& \|v(t)\|_{L_{t}^{r}\left(\left[t_{0}, T\right], M_{p, 1}^{s}\right)} \\
& \leq \varepsilon+\| \int_{t_{0}}^{t}\left(K(t-\tau)\left(\partial_{t t}+I-\Delta\right) v\right. \\
& \quad+e) d \tau \|_{L_{t}^{r}\left(\left[t_{0}, T\right], M_{p, 1}^{s}\right)} \\
& +\left\|\int_{t_{0}}^{t} K(t-\tau) e d \tau\right\|_{L_{t}^{r}\left(\left[t_{0}, T\right], M_{p, 1}^{s}\right)} \\
& \leq \varepsilon+\left\|\left(\partial_{t t}+I-\Delta\right) v+e\right\|_{L_{t}^{r /(k+1)}\left(\left[t_{0}, T\right], M_{p^{\prime}, 1}^{s}\right)} \\
& +\|e\|_{L_{t}^{r /(k+1)}\left(\left[t_{0}, T\right], M_{p^{\prime}, 1}^{s}\right)} \\
& \leq \varepsilon+S(T) .
\end{aligned}
$$

By Product Lemma 21, we deduce

$$
\begin{aligned}
S(T)= & \|f(\tilde{u}+v)-f(\widetilde{u})\|_{L_{t}^{r /(k+1)}\left(\left[t_{0}, T\right], M_{p^{\prime}, 1}^{s}\right)} \\
\leq & \|v(t)\|_{L_{t}^{r}\left(\left[t_{0}, T\right], M_{p, 1}^{s}\right)}^{k+1}+\|v(t)\|_{L_{t}^{r}\left(\left[t_{0}, T\right], M_{p, 1}^{s}\right)}^{k} \\
& \times\|\widetilde{u}(t)\|_{L_{t}^{r}\left(\left[t_{0}, T\right], M_{p, 1}^{s}\right)} \\
& +\cdots+\|v(t)\|_{L_{t}^{r}\left(\left[t_{0}, T\right], M_{p, 1}^{s}\right)} \\
& \times\|\widetilde{u}(t)\|_{L_{t}^{r}\left(\left[t_{0}, T\right], M_{p, 1}^{s}\right)}^{k} .
\end{aligned}
$$

Noticing that $\|\widetilde{u}(t)\|_{L_{t}^{r}\left(\left[t_{0}, T\right], M_{p, 1}^{s}\right)} \leq \varepsilon_{c}$, we have

$$
\begin{aligned}
S(T) \lesssim & (\varepsilon+S(T))^{k+1}+(\varepsilon+S(T))^{k} \varepsilon_{c} \\
& +\cdots+(\varepsilon+S(T)) \varepsilon_{c}^{k}
\end{aligned}
$$

for every $T \in I$.
A standard continuity argument then shows that if we shrink $\varepsilon_{c}$ sufficiently small, then

$$
S(T) \lesssim \varepsilon
$$

for any $T \in I$, which implies (23). Using (124) and (127), one can easily obtain (24). By Lemma 19, we have

$$
\begin{gathered}
\left\|K^{\prime}\left(t-t_{0}\right) v\left(t_{0}\right)+K\left(t-t_{0}\right) v_{t}\left(t_{0}\right)\right\|_{L_{t}^{\infty}\left(I, M_{p, 1}^{s}\right)} \\
\lesssim C(I)\left(\left\|u\left(t_{0}\right)-\widetilde{u}\left(t_{0}\right)\right\|_{M_{p, 1}^{s}}\right. \\
\left.+\left\|u_{t}\left(t_{0}\right)-\widetilde{u}_{t}\left(t_{0}\right)\right\|_{M_{p, 1}^{s-1}}\right) \\
\lesssim C(I)\left(M^{\prime}+\widetilde{M^{\prime}}\right) \\
\left\|-J^{2} K\left(t-t_{0}\right) v\left(t_{0}\right)+K^{\prime}\left(t-t_{0}\right) v_{t}\left(t_{0}\right)\right\|_{L_{t}^{\infty}\left(I, M_{p, 1}^{s-1}\right)} \\
\lesssim C(I)\left(M^{\prime}+\widetilde{M^{\prime}}\right) .
\end{gathered}
$$

By Lemma 20 and Hölder inequality, we have that for every $t \in I$

$$
\begin{gathered}
\left\|\int_{t_{0}}^{t} K(t-\tau)(f(\tilde{u}+v)-f(\widetilde{u})) d \tau\right\|_{M_{p, 1}^{s}} \\
\lesssim \int_{I}(1+|t-\tau|)^{-n(1 / 2-1 / p)} \\
\quad \times\|f(\widetilde{u}+v)-f(\widetilde{u})\|_{M_{p^{\prime}, 1}^{s}} d \tau \\
\lesssim \int_{I}\|f(\widetilde{u}+v)-f(\widetilde{u})\|_{M_{p, 1}^{s}} d \tau \\
\left.\lesssim|I|^{\beta}\|f(\widetilde{u}+v)-f(\widetilde{u})\|_{L_{t}^{r /(k+1)}\left(I, M_{p^{\prime}, 1}^{s}\right.}\right) \\
\lesssim C(I) \varepsilon .
\end{gathered}
$$

Similarly, we have

$$
\begin{gathered}
\left\|\int_{t_{0}}^{t} K^{\prime}(t-\tau)(f(\tilde{u}+v)-f(\tilde{u})) d \tau\right\|_{M_{p, 1}^{s-1}} \lesssim C(I) \varepsilon, \\
\left\|\int_{t_{0}}^{t} K(t-\tau) e d \tau\right\|_{L_{t}^{\infty}\left(I, M_{p, 1}^{s}\right)} \lesssim C(I) \varepsilon, \\
\left\|\int_{t_{0}}^{t} K^{\prime}(t-\tau) e d \tau\right\|_{L_{t}^{\infty}\left(I, M_{p, 1}^{s-1}\right)} \lesssim C(I) \varepsilon,
\end{gathered}
$$

and then we use the integral equation to conclude (25) and (26). Finally, (27) and (28) are easy conclusions from (25), (26), and (20).

After proof of short-time perturbations, we will derive long-time perturbations by an iterative procedure. In longtime perturbations, the smallness condition $\|\widetilde{u}\|_{L_{t}^{r}\left(I, M_{p, 1}^{s}\right)} \leq \varepsilon_{c}$ will be replaced by a bound condition $\|\widetilde{u}\|_{L_{t}^{r}\left(I, M_{p, 1}^{s}\right)} \leq L$. In our proof, we need to divide interval $I$ into several subintervals $I_{j}$ to gain a small size of $\|\widetilde{u}\|_{L_{t}^{r}\left(I_{j}, M_{p, 1}^{s}\right)}$. The $k$-admissible condition which ensures $r<\infty$ allows us to do so. 
5.2. Proof of Long-Time Perturbations. For convenience, we also assume that $t_{0}=\inf I$. We subdivide $I$ into $N$ subintervals $I_{j}=\left[t_{j}, t_{j+1}\right], j=0, \ldots, N-1$ such that

$$
\|\tilde{u}\|_{L_{t}^{r}\left(I_{j}, M_{p, 1}^{s}\right)} \leq \varepsilon_{c},
$$

where $\varepsilon_{c}$ is as in Theorem 11. Choose $\varepsilon_{1} \leq \varepsilon_{c}$ in Theorem 12 and apply short-time perturbations on $I_{0}$ to obtain

$$
\begin{gathered}
\left\|\left(\partial_{t t}+I-\Delta\right)(u-\widetilde{u})+e\right\|_{L_{t}^{r /(k+1)}\left(I_{0}, M_{p^{\prime}, 1}^{s}\right)} \leqslant \varepsilon, \\
\|u-\widetilde{u}\|_{L_{t}^{r}\left(I_{0}, M_{p, 1}^{s}\right)} \lesssim \varepsilon .
\end{gathered}
$$

Using the integral equation on $I_{1}$, we have

$$
\begin{aligned}
\| K^{\prime}(t & \left.-t_{1}\right) v\left(t_{1}\right)+K\left(t-t_{1}\right) v_{t}\left(t_{1}\right) \|_{L_{t}^{r}\left(I_{1}, M_{p, 1}^{s}\right)} \\
\lesssim & \left\|K^{\prime}\left(t-t_{0}\right) v\left(t_{1}\right)-K\left(t-t_{0}\right) v_{t}\left(t_{1}\right)\right\|_{L_{t}^{r}\left(I_{1}, M_{p, 1}^{s}\right)} \\
& +\left\|\left(\partial_{t t}+I-\Delta\right) v+e\right\|_{L_{t}^{r /(k+1)}\left(\left[t_{0}, t_{1}\right], M_{p^{\prime}, 1}^{s}\right)} \\
& +\|e\|_{L_{t}^{r /(k+1)}\left(\left[t_{0}, t_{1}\right], M_{p^{\prime}, 1}^{s}\right)} \\
& \leq \varepsilon+\varepsilon+\varepsilon .
\end{aligned}
$$

So we can shrink $\varepsilon_{1}$ such that

$$
\left\|K^{\prime}\left(t-t_{1}\right) v\left(t_{1}\right)+K\left(t-t_{1}\right) v_{t}\left(t_{1}\right)\right\|_{L_{t}^{r}\left(I, M_{p, 1}^{s}\right)} \leq \varepsilon_{c} .
$$

We also have

$$
\|e\|_{L_{t}^{r /(k+1)}\left(I_{1}, M_{p^{\prime}, 1}^{s}\right)} \leq\|e\|_{L_{t}^{r /(k+1)}\left(I, M_{p^{\prime}, 1}^{s}\right)} \leq \varepsilon \leq \varepsilon_{c} .
$$

So we can use the short-time perturbations to obtain

$$
\begin{gathered}
\left\|\left(\partial_{t t}+I-\Delta\right)(u-\widetilde{u})+e\right\|_{L_{t}^{r /(k+1)}\left(I_{1}, M_{p^{\prime}, 1}^{s}\right)} \lesssim C(1) \varepsilon, \\
\|u-\widetilde{u}\|_{L_{t}^{r}\left(I_{1}, M_{p, 1}^{s}\right)} \lesssim C(1) \varepsilon .
\end{gathered}
$$

For $I_{2}$, repeat the process above to shrink $\varepsilon_{1}$ to continue the inductive argument. Accordingly, after a finite number of "shrinks" of $\varepsilon_{1}$, we have, for all $m \in[1, N-1]$,

$$
\left\|K^{\prime}\left(t-t_{m}\right) v\left(t_{m}\right)+K\left(t-t_{m}\right) v_{t}\left(t_{m}\right)\right\|_{L_{t}^{r}\left(I, M_{p, 1}^{s}\right)}<\varepsilon
$$

and then, for some $0<\varepsilon<\varepsilon_{1}$ and $0 \leq j \leq J$, we have

$$
\begin{gathered}
\left\|\left(\partial_{t t}+I-\Delta\right)(u-\widetilde{u})+e\right\|_{L_{t}^{r /(k+1)}\left(I_{j}, M_{p^{\prime}, 1}^{s}\right)} \leqslant C(j) \varepsilon, \\
\|u-\widetilde{u}\|_{L_{t}^{r}\left(I_{j}, M_{p, 1}^{s}\right)} \lesssim C(j) \varepsilon,
\end{gathered}
$$

where $C(j)$ only depends on $j$. So we have

$$
\begin{aligned}
\|\left(\partial_{t t}+\right. & I-\Delta)(u-\tilde{u})+e \|_{L_{t}^{r /(k+1)}\left(I, M_{p^{\prime}, 1}^{s}\right)} \\
& \lesssim \sum_{j=0}^{N-1} C(j) \varepsilon \lesssim C(L) \varepsilon .
\end{aligned}
$$

By smallness assumption, we also have

$$
\|u-\widetilde{u}\|_{L_{t}^{r}\left(I, M_{p, 1}^{s}\right)} \leqslant C(L) \varepsilon .
$$

Using integral equation

$$
\begin{aligned}
v(t)= & K^{\prime}\left(t-t_{0}\right) v\left(t_{0}\right)+K\left(t-t_{0}\right) v_{t}\left(t_{0}\right) \\
& -\int_{t_{0}}^{t} K(t-\tau)(f(\tilde{u}+v)-f(\widetilde{u})-e) d \tau
\end{aligned}
$$

and Hölder inequality, we conclude that

$$
\begin{aligned}
& \|v(t)\|_{L_{t}^{\infty}\left(I, M_{p, 1}^{s}\right)} \\
& \leq C(|I|)\left(\left\|v\left(t_{0}\right)\right\|_{M_{p, 1}^{s}}+\left\|v_{t}\left(t_{0}\right)\right\|_{M_{p, 1}^{s-1}}\right) \\
& +C(|I|)\left(\|f(\widetilde{u}+v)-f(\widetilde{u})\|_{L_{t}^{r /(k+1)}\left(I, M_{p^{\prime}, 1}^{s}\right)}\right. \\
& \left.+\|e\|_{L_{t}^{r /(k+1)}\left(I, M_{p^{\prime}, 1}^{s}\right)}\right) \\
& \leq C(|I|, L)\left(M^{\prime}+\widetilde{M^{\prime}}+\varepsilon\right) .
\end{aligned}
$$

In the same way, we have

$$
\left\|v_{t}(t)\right\|_{L_{t}^{\infty}\left(I, M_{p, 1}^{s-1}\right)} \lesssim C(|I|, L)\left(\widetilde{M^{\prime}}+M^{\prime}+\varepsilon\right) .
$$

Finally, we can use (29) to deduce (37) and (38).

5.3. Proof of Continuous Dependence. Since $1 /(k+2) \leq 1 / p<$ $1 / 2-1 / n(k+1),(p, k+1)$ is a $k$-admissible pair. For every compact interval $I \subset\left(T_{\min }\left(u_{0}\right), T_{\max }\left(u_{0}\right)\right)$, noticing that

$$
\begin{gathered}
\left\|K^{\prime}\left(t-t_{0}\right)\left(u_{0, n}-u_{0}\right)+K\left(t-t_{0}\right)\left(u_{1, n}-u_{1}\right)\right\|_{L_{t}^{k+1}\left(I, M_{p, 1}^{s}\right)} \\
\quad \lesssim C(|I|)\left(\left\|u_{0, n}-u_{0}\right\|_{M_{p, 1}^{s}}+\left\|u_{1, n}-u_{1}\right\|_{M_{p, 1}^{s-1}}\right),
\end{gathered}
$$

we apply long-time perturbations with $r=k+1$ and $e=0$ to conclude that there exists a small constant $\varepsilon_{2}=$ $\varepsilon_{2}\left(|I|,\|u\|_{L_{t}^{k+1}\left(I, M_{p, 1}^{s}\right)}\right)$ such that if

$$
\left\|u_{0, n}-u_{0}\right\|_{M_{p, 1}^{s}}+\left\|u_{1, n}-u_{1}\right\|_{M_{p, 1}^{s-1}} \leq \varepsilon_{2},
$$

then (1) has a strong solution $u_{n}$ on $I$ with the initial data $u_{n}\left(t_{0}\right)=u_{0, n}$ and

$$
\left\|u_{n}-u\right\|_{L_{t}^{\infty}\left(I, M_{p, 1}^{s}\right)} \lesssim C\left(|I|,\|u\|_{L_{t}^{k+1}\left(I, M_{p, 1}^{s}\right)}\right) \varepsilon_{2} .
$$

So is $\left\|u_{n}-u\right\|_{L_{t}^{\infty}\left(I, M_{p, 1}^{s-1}\right)}$.

The proof of Corollary 14 is similar, and we omit it here.

\section{Conflict of Interests}

The authors declare that there is no conflict of interests regarding the publication of this paper.

\section{Acknowledgments}

This work is partially supported by the NSF of China (Grant no. 11271330) and PSF of Zhejiang Province (BSH 1302046). 


\section{References}

[1] M. Ruzhansky, M. Sugimoto, and B. X. Wang, "Modulation spaces and nonlinear evolution equations," Progress in Mathematics, vol. 301, pp. 267-283, 2012.

[2] B. X. Wang and H. Hudzik, "The global Cauchy problem for the NLS and NLKG with small rough data," Journal of Differential Equations, vol. 232, no. 1, pp. 36-73, 2007.

[3] W. Guo and J. Chen, "Stability of schrödinger equations on modulation spaces," Frontiers of Mathematics in China, vol. 9, no. 2, pp. 275-301, 2014.

[4] Á. Bényi and K. A. Okoudjou, "Local well-posedness of nonlinear dispersive equations on modulation spaces," Bulletin of the London Mathematical Society, vol. 41, no. 3, pp. 549-558, 2009.

[5] T. Tao, "Nonlinear dispersive equations: local and global analysis," CBMS Regional Conference Series in Mathematics 106.

[6] H. G. Feichtinger, "Modulation spaces on locally compact Abelian group," Tech. Rep., University of Vienna, 1983, Published in: "Proceedings of the International Conference on Wavelet and Applications", 99-140. New Delhi Allied, New Delhi, India, 2003.

[7] B. X. Wang, L. F. Zhao, and B. L. Guo, "Isometric decomposition operators, function spaces $E_{p, q}^{\lambda}$ and applications to nonlinear evolution equations," Journal of Functional Analysis, vol. 233, no. 1, pp. 1-39, 2006.

[8] H. Triebel, Theory of Function Spaces, Birkhauser, Basel, Switzerland, 1983.

[9] Á. Bényi, K. Gröchenig, K. A. Okoudjou, and L. G. Rogers, "Unimodular Fourier multipliers for modulation spaces," Journal of Functional Analysis, vol. 246, no. 2, pp. 366-384, 2007.

[10] B. Wang, C. Hao, and C. Huo, Harmonic Analysis Method For Nonlinear Evolution Equations I, World Scientific, Hackensack, NJ, USA, 2011.

[11] J. Chen and D. Fan, "Estimates for wave and Klein-Gordon equations on modulation sapces," Science China Mathematics, vol. 55, no. 10, pp. 2109-2123, 2012.

[12] W. A. Strauss, "Nonlinear scattering theory at low energy," Journal of Functional Analysis, vol. 41, no. 1, pp. 110-133, 1981. 


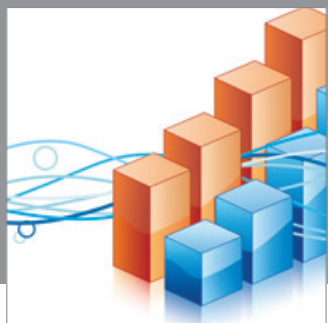

Advances in

Operations Research

mansans

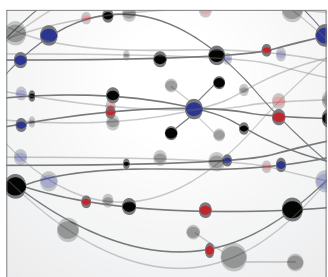

The Scientific World Journal
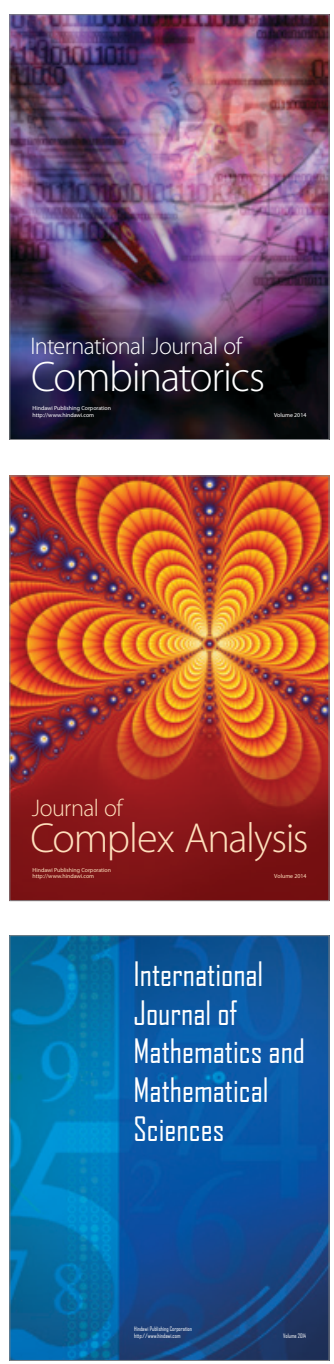
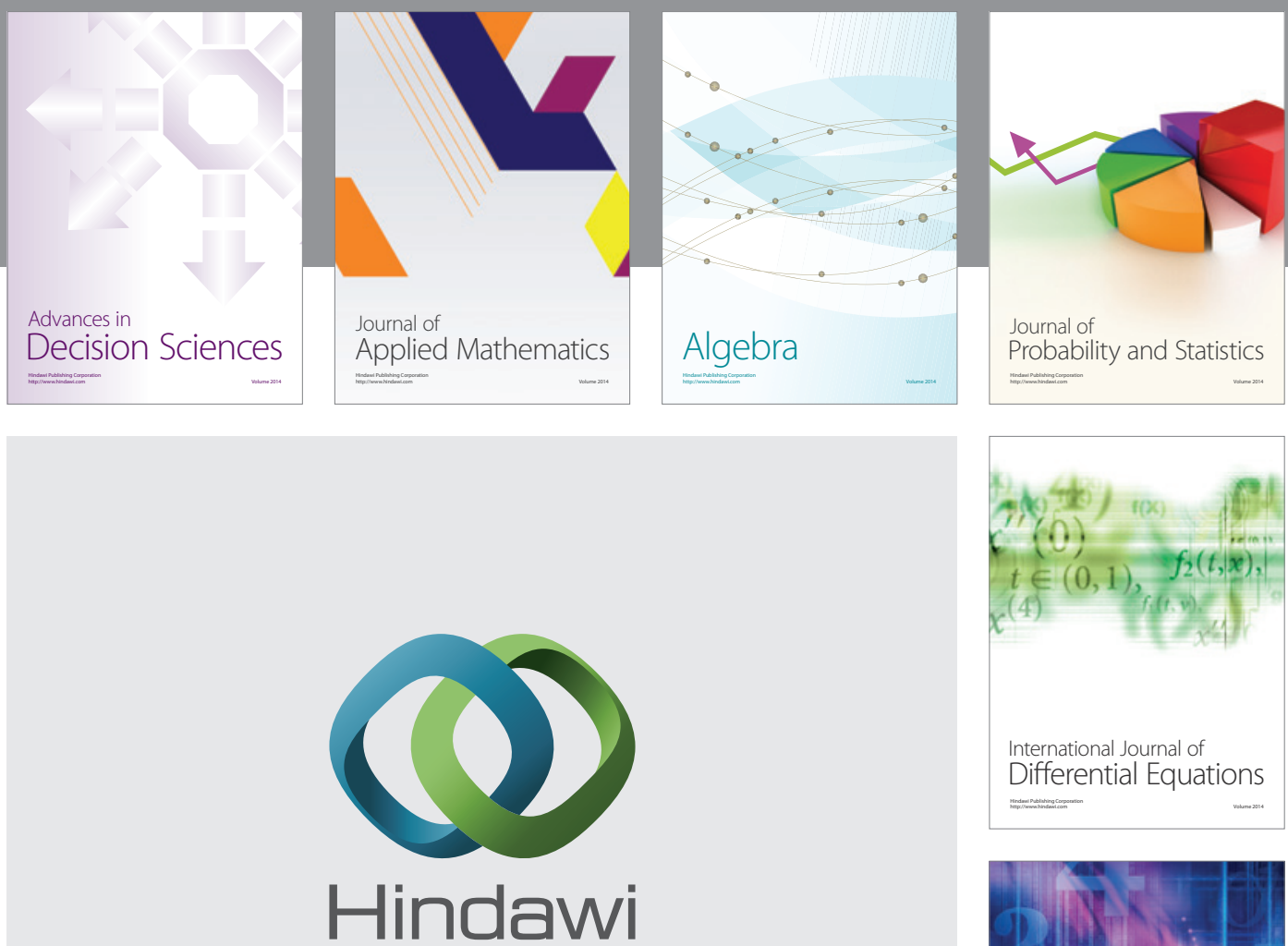

Submit your manuscripts at http://www.hindawi.com
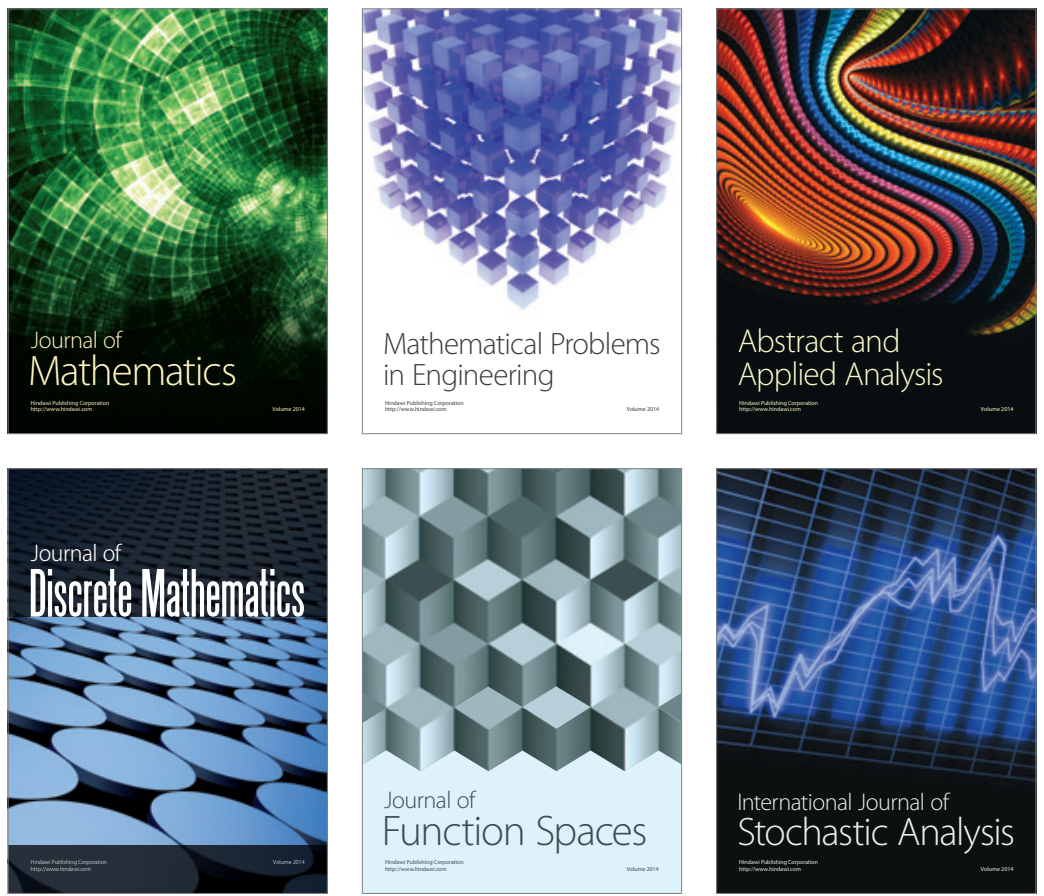

Journal of

Function Spaces

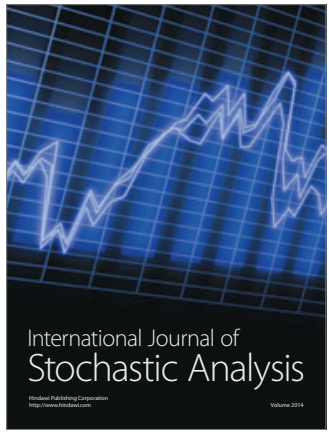

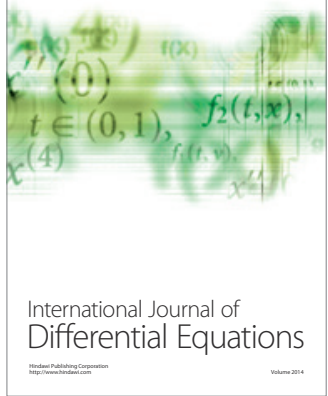
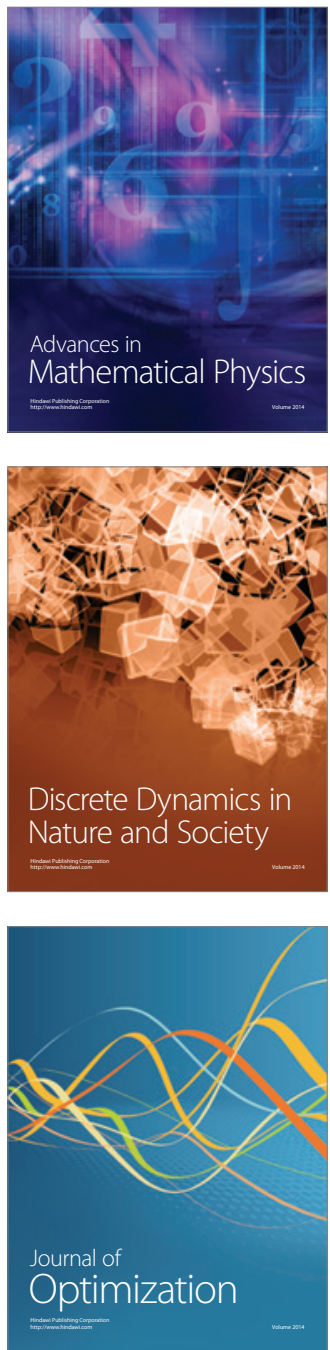